\title{
A new mouse model to study restoration of interleukin-6 (IL-6) expression in a Cre- dependent manner: microglial IL-6 regulation of experimental autoimmune encephalomyelitis
}

Paula Sanchis $^{1 \dagger}{ }^{1+}$ Olaya Fernández-Gayol ${ }^{1,2 \dagger}$, Gemma Comes $^{1}$, Kevin Aguilar ${ }^{1}$, Anna Escrig ${ }^{1}$, Mercedes Giralt ${ }^{1}$, Richard D. Palmiter ${ }^{3}$ and Juan Hidalgo ${ }^{1 *}$ (D)

\begin{abstract}
Background: Interleukin-6 (IL-6) is a pleiotropic cytokine that controls numerous physiological processes both in basal and neuroinflammatory conditions, including the inflammatory response to experimental autoimmune encephalomyelitis (EAE). IL-6 is produced by multiple peripheral and central cells, and until now, the putative roles of IL-6 from different cell types have been evaluated through conditional cell-specific IL-6 knockout mice. Nevertheless, these mice probably undergo compensatory responses of IL-6 from other cells, which makes it difficult to assess the role of each source of IL-6.

Methods: To give some insight into this problem, we have produced a novel mouse model: a conditional reversible IL-6 KO mouse (IL6-DIO-KO). By using double-inverted, open-reading-frame (DIO) technology, we created a mouse line with the loss of 116 expression in all cells that can be restored by the action of Cre recombinase. Since microglia are one of the most important sources and targets of IL-6 into the central nervous system, we have recovered microglial $1 / 6$ expression in IL6-DIO-KO mice through breeding to CX3cr1-CreER mice and subsequent injection of tamoxifen (TAM) when mice were 10-16 weeks old. Then, they were immunized with myelin oligodendrocyte glycoprotein 35-55 peptide $\left(\mathrm{MOG}_{35-55}\right) 7$ weeks after TAM treatment to induce EAE. Clinical symptoms and demyelination, CD3 infiltration, and gliosis in the spinal cord were evaluated.

Results: IL6-DIO-KO mice were resistant to EAE, validating the new model. Restoration of microglial I/6 was sufficient to develop a mild version of EAE-related clinical symptoms and neuropathology.

(Continued on next page)
\end{abstract}

\footnotetext{
* Correspondence: Juan.Hidalgo@uab.es

†Paula Sanchis and Olaya Fernández-Gayol contributed equally to this work. 'Institute of Neurosciences and Department of Cellular Biology, Physiology and Immunology, Animal Physiology Unit, Faculty of Biosciences, Universitat Autònoma de Barcelona, 08193 Barcelona, Spain

Full list of author information is available at the end of the article
}

(c) The Author(s). 2020 Open Access This article is licensed under a Creative Commons Attribution 4.0 International License, which permits use, sharing, adaptation, distribution and reproduction in any medium or format, as long as you give appropriate credit to the original author(s) and the source, provide a link to the Creative Commons licence, and indicate if changes were made. The images or other third party material in this article are included in the article's Creative Commons licence, unless indicated otherwise in a credit line to the material. If material is not included in the article's Creative Commons licence and your intended use is not permitted by statutory regulation or exceeds the permitted use, you will need to obtain permission directly from the copyright holder. To view a copy of this licence, visit http://creativecommons.org/licenses/by/4.0/ The Creative Commons Public Domain Dedication waiver (http://creativecommons.org/publicdomain/zero/1.0/) applies to the data made available in this article, unless otherwise stated in a credit line to the data. 
(Continued from previous page)

Conclusions: IL6-DIO-KO mouse is an excellent model to understand in detail the role of specific cellular sources of IL-6 within a recovery-of-function paradigm in EAE.

Keywords: DIO technology, Conditional reversible IL-6 knockout, Experimental autoimmune encephalomyelitis, Microglial IL-6

\section{Introduction}

Since their inception in the early 80 s, genetically engineered mice have been a very powerful tool in biomedical research [1]. With time, the technology has been refined to allow for conditional knock-out of genes in specific cell populations [2] or in a timed manner [3]. However, the reversibility of these knockouts to allow for recovery of function experiments has been limited and somewhat flawed. Therefore, in this paper, we introduce a conditional (Cre-dependent) and reversible knockout for interleukin-6 (IL-6) using the doubleinverted open reading frame (DIO) paradigm [4]. Our gene of choice for this proof-of-concept knockout was IL-6 since we had already successfully created a floxed mouse to achieve a conditional knockout [5]. The new mouse (IL6-DIO-KO) lacks IL-6 production in all cells, which can be turned back on by the action of Cre recombinase. In this paper, we demonstrate the knockout nature of this mouse in its initial form and test its applicability to specific research questions. We show the effect of selective recovery of IL-6 expression in microglia by crossing IL6-DIO-KO with Cx3cr1-CreER mice $[6,7]$ in the context of experimental autoimmune encephalomyelitis (EAE), the most popular mouse model of multiple sclerosis (MS).

IL-6 is a pleiotropic and multifunctional cytokine that mediates numerous physiological processes and is classically involved in acute and chronic inflammatory conditions [8]. Indeed, its overproduction has been reported in many pathological situations, including MS [9]. In the nervous parenchyma of patients with MS, IL-6 is predominantly linked to the active CNS plaques and located within resident glial cells concentrated in demyelinated areas [10]. IL-6 is also essential in the pathogenesis of EAE, as evidenced by IL- $6 \mathrm{KO}$ mice being resistant to it [11-14] and displaying altered markers of the disease such as reduced infiltration of $\mathrm{T}$ lymphocytes and monocytes into the central nervous system (CNS), reduced expression of adhesion molecules in endothelial cells, as well as defective differentiation of Th17 lymphocytes, which have been proposed as key factors leading to EAE resistance $[11,15]$. In this context, and knowing that IL6 regulates EAE in a source-specific way, given the varied results with conditional cell-specific IL-6 KO mice [16-19], we chose microglia as the cell population to test our reversible KO. Microglial cells have been described as one of the most important IL-6-producing brain cells in neuroinflammatory conditions and are clearly implicated in MS and EAE pathogenesis [20-23].

Our results show that this new IL6-DIO-KO mouse model is a useful tool to easily investigate the specific functions of IL- 6 from each cellular source in EAE pathology. In particular, in the case of recovery of microglial IL-6, this was sufficient to initiate mild paralyzing symptoms related to EAE and slightly regulate the inflammatory cascade of EAE.

\section{Material and methods Animals}

Mice were kept under constant temperature and a 12-h light:12-h dark with food and water available ad libitum. All experiments were approved by the Ethics Committee on Animal Experiments of the Universitat Autònoma de Barcelona and the Generalitat de Catalunya (Refs. 3782 and 9684, respectively).

\section{Generation of IL6-DIO-KO mice}

The conditional reversible IL-6 KO (IL6-DIO-KO) mice were developed using double-inverted open reading frame (DIO) technology [4]. Exon 2 of the Il6 gene $\left(I l 6^{\text {DIOex2 }}\right.$ ) was inverted and flanked by two pairs of mutually incompatible loxP sites (lox2272 and loxP) in opposite orientations resulting in an inactive $I l 6$ allele. The action of Cre recombinase inverts exon 2 thereby restoring normal Il6 expression.

This mouse was generated as follows: a BAC clone from a $\mathrm{C} 57 \mathrm{Bl} / 6 \mathrm{~J}$ mouse containing the $I l 6$ sequence was obtained from Invitrogen (RP23-121 M2). A $5614 \mathrm{bp}$ fragment with engineered HpaI and PacI sites upstream of exon 2 and a $5241 \mathrm{bp}$ fragment with engineered XhoI and NotI sites downstream of exon 2 of the Il6 gene were synthesized by PCR (using Q5 polymerase, NE Biolabs) (Fig. 1a, left). Exon 2 flanked by double lox sites with PacI and SalI sites at the ends was synthesized by Genscript and inserted into a targeting vector in the reverse orientation. The three fragments were sequentially cloned into a targeting vector containing Pgk-DTa and $H S V-T K$ genes for negative selection and frt-flanked $S v N e o R$ for positive selection. The final construct was purified from a $\mathrm{CsCl}$ gradient, linearized with $A s c I$ and electroporated into G4 hybrid (C57Bl/6 × Sv129) embryonic stem (ES) cells (Fig. 1a, right). Correctly targeted 


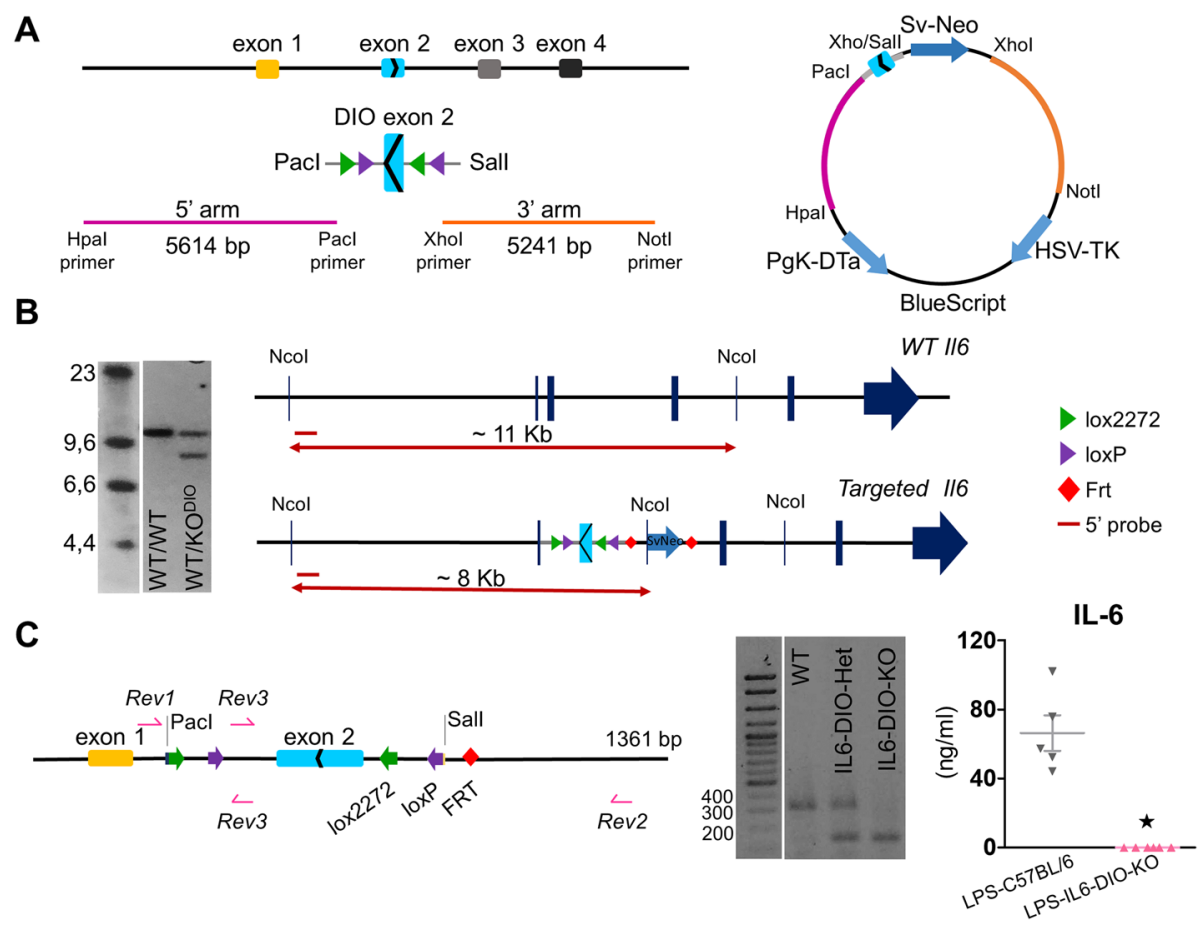

Fig. 1 Generation of a conditional reversible KO mouse for IL-6. a Outline of the targeting strategy. Left: 5614 bp Hpal-Pacl (pink; upstream) and a 5241 bp Xhol-Notl (orange; downstream) flanking $1 / 6^{D / 0 e x 2}$ fragments. Right: the final product that consists of a Pacl-Sall fragment with inverted exon 2 flanked by double lox sites, and three fragments containing Pgk-DTa and HSV-TK genes for negative selection and frt-flanked SvNeo gene for positive selection. $\mathbf{b}$ Southern blot for 116 gene of the positive ES clones using the probe (left) revealed a $\sim 11 \mathrm{~kb}$ band on the wild type allele and $\mathrm{a} \sim 8 \mathrm{~kb}$ band on the targeted allele, as expected (right); those clones with two bands were selected. c Left: The $1 / 6$ gene-modified sequence from IL6-DIO-KO mouse was sequenced by the Sanger method. The resulting sequence had 1361 bp and exon 1 (yellow), exon 2 (blue), lox2272 cassettes (green), loxP cassettes (purple), and FRT (red) were genetic structures observed in that sequence. Middle: PCR products for II6 gene of WT, IL6-DIO-Het, and IL6-DIO-KO mice yielded the expected bands. Right: IL-6 protein in serum was undetectable by ELISA in IL6-DIO-KO mice after LPS administration. All results are represented as mean \pm SEM; $\star p \leq 0.05$ vs. LPS-C57BL/6 mice

clones were identified by Southern blot; approximately $5 \mu \mathrm{g}$ of DNA from ES cells were digested with NcoI, electrophoresed on a $0.7 \%$ agarose gel and transferred to a nylon membrane. Next, the membrane was hybridized to a unique probe of $621 \mathrm{bp}$ located outside the $5^{\prime}$ arm (synthesized using the primers $F w$ : 5'-CAGCATCTCA TCTGAGTTCCG-3' and Rv: 5'-CTCACTGTTC ACAAAGCACAGG-3') (Additional file 1) that would give $\mathrm{a} \sim 11 \mathrm{~kb}$ band for the wild-type allele and a $\sim 8 \mathrm{~kb}$ band for the targeted allele (Fig. 1b). Clones with the two bands were reassessed using $\mathrm{Neo}$ as a probe. Four positive clones (out of 81 analyzed) were correctly targeted and had a single NeoR gene insert. Three of those were injected into $\mathrm{C} 57 \mathrm{Bl} / 6$ blastocysts, which were implanted in receptive females. Those mice with a high percentage of chimerism were then crossed with Gt(RO$S A) 26 S o r-F L P$ recombinase to remove the Sv-NeoR gene [24]. Mice heterozygous for the $I l 6^{\text {DIOex2 }}$ gene were backcrossed >10 times to $\mathrm{C} 57 \mathrm{BL} / 6 \mathrm{OlaHsd}$ mice, and non-littermate heterozygous mice for $I l 6^{\text {DIOex2 }}$ gene from the tenth crossing were crossed to each other to obtain three possible genotypes: $I l 6^{\text {DIOex2/DIOex2 }}$ (IL6-
DIO-KO mice), $I l 6^{\mathrm{WT} / \mathrm{WT}}$ (WT mice) and $I l 6^{\mathrm{DIOex} 2 / \mathrm{WT}}$ (IL6-DIO-Het mice, with one functional Il6 allele).

\section{Reactivation of microglia-derived II6 expression}

IL6-DIO-KO mice were crossed with heterozygous Cx3cr1-CreER mice, produced by Drs. Jung and Prinz and obtained from the European Mouse Mutant Archive (EMMA) repository, where were backcrossed with C57BL/6 mice more than 10 generations [7]. Then, the offspring Cre-positive animals from the first crossing were selected and crossed again with homozygous IL6DIO-KO mice to obtain mice homozygous for IL6-DIO$\mathrm{KO}$ and carrying $\mathrm{Cx} 3 \mathrm{cr} 1-\mathrm{CreER}$ allele; these mice are designated IL6-DIO-ON ${ }^{\mathrm{Cx} 3 \mathrm{cr} 1}$ and should have Il6 expression only where $\mathrm{Cx} 3 \mathrm{cr} 1$ gene is expressed, including microglia.

\section{Genotyping}

Mice were genotyped by PCR analysis of tail and liver DNA, extracted previously by boiling in $100 \mu \mathrm{l}$ of $50 \mathrm{mM}$ sodium hydroxide for $7 \mathrm{~min}$, using three primers (Rev1: 5'-GAGACTGTGAGAGAGGAGTGTG-3'; Rev2: 5'- 
CATCTTATCTGGGCTGACCCTAG-3'; Rev3: 5'-TCTC TGCTGGGATCTAGGGCC-3') and the following reaction conditions: $95^{\circ} \mathrm{C} 2 \mathrm{~min}$, followed by 30 cycles of $95^{\circ} \mathrm{C} 30 \mathrm{~s}, 52^{\circ} \mathrm{C} 30 \mathrm{~s}$, and $72{ }^{\circ} \mathrm{C} 30 \mathrm{~s}$, and $72{ }^{\circ} \mathrm{C} 5 \mathrm{~min}$. Finally, Cre primers and PCR conditions were adapted from Sanz et al. [25].

\section{Sanger DNA sequencing}

The modified fragment of $I l 6$ gene from IL6-DIO-KO mouse was sequenced by the Sanger method. Two PCR products were amplified separately using two pairs of primers. The first pair of primers amplified from the middle of the first exon to the end of the third intron (1F: 5'-CCCACCAAGAACGATAGTCA-3' and $\triangle R$ : 5'ATGCCCAGCCTAATCTAGGT-3' [5] while the second pair amplified from the first intron to the end of the second intron (fw: 5'-CGATGCTAAACGACGTCACA3'and Rev3 primer). Then, the PCR fragments were purified by ExoSAP-IT Express PCR Cleanup Reagent (ThermoFisher, 75001). The enzymatic cleanup method and sequencing were performed by Servei de Genòmica i Bioinformàtica (IBB-UAB). The data were imported firstly into Snapgene Viewer 4.3.4. Software (from Insightful Science; available at snapgene.com), in which both sequences were overlapped creating a unique sequence with $1361 \mathrm{bp}$.

\section{Tamoxifen treatment}

Cx3cr1-CreER mouse is a tamoxifen (TAM)-inducible model [7], then the IL6-DIO-ON ${ }^{\mathrm{Cx} 3 \mathrm{cr} 1}$ mice and their littermates were injected for five or eleven consecutive days with TAM. Their littermates were also administered with TAM to control the effects of TAM in the analyzed parameters. TAM (Sigma 5648) was dissolved in ethanol at a concentration of $10 \mathrm{mg} / 100 \mu \mathrm{l}$ by shaking and heating at $37^{\circ} \mathrm{C}$ [26]; next, the solution was diluted with sunflower oil to $10 \mathrm{mg} / \mathrm{ml}$ and one daily dose of $100 \mu \mathrm{l}(1 \mathrm{mg})$ was injected intraperitoneally to each mouse.

\section{Lipopolysaccharide injection}

Mice were injected intraperitoneally with $0.5 \mathrm{mg} / \mathrm{Kg}$ lipopolysaccharide (LPS O55:B5, Sigma L2880) and blood was drawn from the tail $90 \mathrm{~min}$ later; it was centrifuged at $9.500 \mathrm{xg}$ for $10 \mathrm{~min}$ at $4{ }^{\circ} \mathrm{C}$ and serum was stored at $-80^{\circ} \mathrm{C}$ before testing.

\section{Microglia isolation}

IL6-DIO-KO and IL6-DIO-ON ${ }^{\mathrm{Cx} 3 \mathrm{cr} 1}$ mice were euthanized 1 month after 11 days of TAM treatment and brains (excluding cerebellum) were quickly dissected and chopped in small pieces. Then, the tissue was dissociated both enzymatically and mechanically using the adult brain dissociation kit (Miltenyi 130-107-677) and placing it on a gentleMACS Octo Dissociator for $30 \mathrm{~min}$ at $37^{\circ} \mathrm{C}$. After dissociation, debris and erythrocytes were removed and microglia were isolated using magnetic microbeads against Cluster of differentiation molecule 11b (CD11b; Miltenyi 130-093-634) following the manufacturer's instructions. The purity of microglia in CD11b+ cell population was previously checked in [26]. The CD11b-negative flow-through was named "remaining sample". DNA from cells was extracted as described above.

\section{Induction of EAE and clinical evaluation}

The induction of EAE was carried out using adult mice. All animals were immunized under isoflurane anesthesia (oxygen flowmeter to $0.8 \mathrm{~L} / \mathrm{min}$ and isoflurane vaporizer to 4 and $1.5 \%$ during induction and maintenance, respectively) as described [19]. On day 0, mice were injected subcutaneously into the hind flanks with an emulsion of $100 \mu \mathrm{L} \quad \mathrm{MOG}_{35-55} \quad(3 \mathrm{mg} / \mathrm{ml}$; sequence: MEVGWYRSPFSRVVHLYRNGK-carboxyl. Purity: $>98 \%$. Peptide synthetized upon request in the Peptide Synthesis Facility at the Universitat Pompeu Fabra) and $100 \mu \mathrm{L}$ of complete Freund's adjuvant (CFA; SigmaAldrich F5881) supplemented with $3 \mathrm{mg} / \mathrm{ml}$ Mycobacterium tuberculosis H37RA (BD Difco 231141). Control mice were immunized with an emulsion containing bovine serum albumin (BSA; $3 \mathrm{mg} / \mathrm{ml}$; Sigma A9647) instead $\mathrm{MOG}_{35-55}$ peptide. All mice received an intraperitoneal injection of $500 \mathrm{ng}$ Bordetella pertussis toxin (Native Antigen Company PT-TNL-50) on day 0 following $\mathrm{MOG}_{35-55} / \mathrm{BSA}$ injection and on day 2 postimmunization. Bordetella pertussis toxin is widely used to facilitate the induction of EAE since it destabilizes the blood-brain barrier and facilitates the migration of $\mathrm{T}$ cells into the CNS.

After immunization, body weight and clinical score were monitored daily. The progression of the disease was assessed in each animal with a numerical scale following these criteria: $0=$ no clinical signs, $0.25=$ slight loss of tail tonus, $0.5=$ partial loss of tail tonus, $1=$ paralyzed tail, $2=$ moderate hind limb paraparesis, $2.5=$ severe hind limb paraparesis, $3=$ partial hind limb paralysis, $3.5=$ hind limb paralysis, $4=$ tetraplegia, and 5 $=$ death. When mice lost more than $10 \%$ weight, they were injected intraperitoneally with $200 \mu \mathrm{l}$ of saline buffer supplemented with $3.6 \%$ glucose. We applied the following endpoint criteria: (i) self-mutilation; (ii) weight loss greater than 25\%; or (iii) tetraplegia for more than 2 days. The time to disease onset and time to peak disease (days to peak score) were defined by a clinical score $\geq 1$. The peak score (highest score) and median score were also calculated individually.

Mice were euthanized by decapitation and their spine was cut between the ninth and tenth thoracic vertebrae. 
The upper part was processed for histological analysis (see "Histology" section) and the lower part was cut open to extract the spinal cord (segments T12-Co3), which was snap-frozen in liquid nitrogen and stored at $-80^{\circ} \mathrm{C}$ for molecular analysis of the inflammatory response. Blood was collected from the trunk and the serum was obtained as mentioned before and stored at $-80^{\circ} \mathrm{C}$.

\section{IL-6 enzyme-linked immunosorbent assays (ELISA)}

IL-6 levels in serum were measured using IL-6 ELISA following manufacturer's instructions (Bionova 860020192).

\section{Real-time polymerase chain reaction (qPCR)}

RNA was extracted using Promega Maxwell RSC simplyRNA kit following the manufacturer's instructions. Then, $2 \mu \mathrm{g}$ of RNA of each sample were treated with an extra DNase I step (Qiagen). After removing DNA from the RNA samples, a two-step qPCR protocol was performed using iScript cDNA synthesis kit (BioRad) and iTaq Universal SYBR Green Supermix (Bio-Rad Laboratories 1725124). Fold change expression was calculated with an adaptation of delta-delta-Ct method using Glyceraldehyde 3-phosphate dehydrogenase (Gapdh) as the reference gene and the EAE IL6-DIO-Het group as calibrator.

\section{Histology}

The upper part of the vertebral column with the spinal cord from sham and $\mathrm{MOG}_{35-55}$-immunized mice was fixed with $4 \%$ PFA for $24 \mathrm{~h}$. Afterwards, they were carefully dissected, being the spinal cord post-fixed for another $24 \mathrm{~h}$ in $4 \%$ PFA, subsequently embedded in paraffin and cut sagittally into $8-\mu \mathrm{m}$-thick sections. Two non-consecutive slices from each animal were used for each histological analysis. For myelin evaluation, samples were stained with $0.1 \%$ Luxol Fast Blue (LFB) overnight at $56{ }^{\circ} \mathrm{C}$. The following day, the tissue was differentiated with $0.05 \%$ lithium carbonate (Fluka, $62 \mathrm{~K} 70$ ) and $70 \%$ ethanol, and counterstained with hematoxylin solution (Sigma MHS16). For IHC analysis, microglia/macrophages, astrocytes and lymphocytes cluster of differentiation $3+(\mathrm{CD} 3+)$ were identified by antibodies against ionized calcium-binding adaptor molecule-1 (IBA-1; Wako 019-19741, 1/1500), Glial fibrillary acidic protein (GFAP; Dako Z0334, 1/900), and CD3+ (Dako A0452, 1/ 100), respectively.

Antigen retrieval was necessary in IBA-1 and CD3 IHC. For the former, samples were treated with $10 \mathrm{mM}$ sodium citrate $0.05 \%$ tween for $20 \mathrm{~min}$ at $96^{\circ} \mathrm{C}$, whereas for the latter, they were incubated with $1 \mathrm{~g} / \mathrm{l}$ protease type XIV (Sigma, P5147) for $8 \mathrm{~min}$ at $37^{\circ} \mathrm{C}$. Then, samples were incubated with endogenous peroxidase blocking buffer $(70 \%$ methanol and 3\% hydrogen peroxide
$\left.\left(\mathrm{H}_{2} \mathrm{O}_{2}\right)\right)$ for 15 min and then blocked in 1\% BSA in 0.05 $\mathrm{M}$ Tris-buffered saline and $0.5 \%$ triton-X100 for $1 \mathrm{~h}$. The incubation with primary antibodies was overnight at $4{ }^{\circ} \mathrm{C}$. The following day, tissues were incubated with a biotin-conjugated secondary antibody Atom BA-1000 $(1 / 300)$ for $1 \mathrm{~h}$ and then, with horseradish peroxidasecoupled streptavidin (Vector SA-5004, 1/500-600). Immunoreactivity was visualized by adding $50 \mathrm{mg} / \mathrm{ml} \mathrm{3}$, 3-diaminobenzidine-tetrahydrochloride and $0.033 \%$ $\mathrm{H}_{2} \mathrm{O}_{2}$ for 1-5 min, depending on the primary antibody. Samples for CD3 were counterstained with hematoxylin solution (Sigma MHS16-500 ml).

Serial images of the tissues from duplicates of each animal were taken at $\times 10$ using a Nikon Eclipse 90i microscope and Nikon Act-1 software. Quantification was performed with ImageJ software [27], using a color deconvolution plugin [28] in CD3 IHC counterstained with hematoxylin and $\mathrm{LFB} /$ hematoxylin staining to separate both colors and quantify only CD3+ cells and myelin, respectively. In LFB staining, the percentage of demyelination was evaluated in the white matter (WM). For CD3 IHC, we quantified the integrated density (sum of all the pixel intensities in the region of interest) of CD3 per infiltrate. In IBA-1 immunostaining, manual count of IBA-1+ cells in the white and gray (GM) matter of the spinal cord was carried out in the first EAE experiment whereas the quantification of the integrated density of white and gray matter, as well as the manual count of IBA-1+ cells in the white matter, were performed in the second EAE experiment. In addition, we characterized IBA-1+ cells in terms of their morphology. IBA-1+ cells with long and thin processes (ramified morphology) were classified as resting IBA-1+ cells whereas those with an enlarged body and small process were considered reactive IBA-1+ cells. IBA-1+ cells with a round body were classified as fully activated cells. In GFAP immunostaining, manual count of GFAP + cells were performed in the GM in the first experiment; however, this was not possible in the white matter given its background was too high. The quantification of the integrated density of white and gray matter, as well as manual count of GFAP + cells in the GM gray matter, were carried out in the second EAE experiment. Furthermore, those cells with thin and reduced processes (ramified morphology) were classified as resting GFAP+ cells whereas those with thicker hypertrophic processes (starshaped morphology) were recorded as reactive GFAP+ cells. All results were normalized to the measured area.

\section{Statistics}

Data is shown as mean \pm SEM, plus individual data points (except for clinical score), except for EAE summary tables, where median and interquartile range (IQR) are used. Statistical calculations were carried out using 
the Statistical Package for Social Sciences software (SPSS, version 19) unless otherwise stated. The clinical score was analyzed with a generalized linear model (GzLM) using the area under the curve (AUC), which had been previously calculated using GraphPad Prism. Body weight gain and incidence were analyzed by generalized estimated equations (GEE) and Fisher's exact test, respectively. The day of onset (with score $\geq 1$ ) and time to peak (with score $\geq 1$ ) were examined using KaplanMeier survival analysis plus log-rang test, and the posthoc pairwise comparisons were performed using the Holm-Bonferroni correction ( $\mathrm{R}$ packages "survival" and "survminer"; RStudio (version 1.2.5033, with $\mathrm{R}$ version 3.6.3)). Peak score and median score were analyzed by Mann-Whitney U test to compare two groups or Kruskal-Wallis rank test and Dunn's test (post hoc) to compare three groups. Histological and gene expression results were analyzed by GzLM using genotype as the main factor and a post hoc sequential Bonferroni test was carried out when the main factor was significant. In the case of comparisons between two groups, the Student $t$ test was used. Outlier detection was carried out using the interquartile range (IQR) rule computed from Tukey's hinges, and values more than 3 IQR's from the end of the box were considered potential outliers and removed from the statistical analysis of histological and gene expression results. Heatmaps to show IL6 levels (or gene expression) alongside AUC were created with the R package "pheatmap" with no clustering and color breaks set with quantiles. Statistical significance was set at $p \leq$ 0.05 for all analyses.

\section{Results}

\section{Generation and characterization of IL6-DIO-KO mice}

We created a new murine model with an inactive $I l 6$ allele (see "Generation of IL6-DIO-KO mice" section) that can be specifically reactivated by the action of Cre recombinase (Fig. 1), using a strategy based on DIO technology [4, 29-31]. In the absence of Cre recombinase, homozygous mice are null for $I l 6$ expression (exon 2 is inverted) and designated as IL6-DIO-KO. The action of Cre recombinase flips exon 2 so that it can be spliced normally thereby restoring Il6 expression; these mice are referred to as IL6-DIO-ON with a superscript indicating what Cre-driver line was used.

We sequenced the modified $I l 6^{\mathrm{DIOex} 2}$ gene of IL6DIO-KO mice by the Sanger method. By using two pairs of primers, we obtained two sequences that were overlapped to create a single genetic map of $1361 \mathrm{bp}$ (Fig. 1c, left and Additional file 2). As expected, 2 pairs of lox cassettes are flanking exon 2 of the Il6 gene on each side (LoxP and Lox2272), two heterologous loxP sites that cannot recombine with each other. In addition, one residual Frt (FLPase recognition target [32];) site is also present. The result of the sequencing showed the inverted exon 2 resulting in an exon that cannot be spliced due to lack of splice acceptor and donor sites.

Three primers were designed for the routine genotyping of the normal and inverted exon 2 (see "Genotyping" section). By using Rev3 and Rev2, a product of $358 \mathrm{bp}$ was amplified for the WT allele of WT and IL6-DIOHet mice, whereas using Rev3 and Rev1 primers, a 193 bp band was obtained for the KO allele of IL6-DIO-Het and IL6-DIO-KO mice (Fig. 1c, middle). Analysis of IL-6 production in IL6-DIO-KO and non-littermate control mice was carried out in serum collected $90 \mathrm{~min}$ after a $0.5 \mathrm{mg} / \mathrm{kg}$ LPS injection. IL-6 protein levels were dramatically increased by LPS in C57BL/6OlaHsd mice, but they remained absent in LPS-IL6-DIO-KO mice (Fig. 1c, right).

IL6-DIO-KO mice and littermate WT controls were immunized with the $\mathrm{MOG}_{33-55}$ peptide, and the clinical course and body weight were monitored for 19 days post-immunization (dpi). MOG-WT mice showed the prototypical course of ascending paralysis starting at $12 \mathrm{dpi}$ (IQR 3.5) and peaking approximately at $15 \mathrm{dpi}$ (IQR 3.5 ) with a clinical score of 3 (IQR 1.6) (Fig. 2a, right and 2b). They also showed a prominent body weight loss in parallel with the appearance of clinical signs (Fig. 2a, left). MOG-IL6DIO-KO mice were resistant to EAE since only $14 \%$ showed minor paralyzing symptoms. The day of disease onset, peak score, time to peak, and median score were all statistically significant between genotypes (Fig. $2 \mathrm{~b}$ and Additional file 3). As expected, IL-6 protein was undetectable in serum samples from MOGIL6-DIO-KO mice, but present in their controls (Fig. 2c). Furthermore, a heatmap of IL-6 levels and area under the curve was performed, reinforcing that the idea that mice with more severe EAE-related symptoms also show more serum IL-6 levels (Fig. 2d).

The upper part of the spinal cord (segments C1-T11) from MOG-WT mice, but not those from MOG-IL6DIO-KO mice, showed clear signs of focal demyelination at $19 \mathrm{dpi}$, in agreement with clinical signs. Infiltration of $\mathrm{CD} 3+\mathrm{T}$ cells was also prominent in the white matter of MOG-WT mice whereas it was practically absent in MOG-IL6-DIO-KO mice (Fig. 3a and b, top). We also counted both the number of GFAP+ and IBA-1+ cells along the spinal cord since astrocytes (GFAP+ cells) and microglia /macrophages (IBA-1+ cells) since they play an important role in EAE pathogenesis mediating the inflammatory response. A more prominent accumulation of GFAP+ cells in the gray matter and IBA-1+ cells both in the white and gray matter were seen in spinal cords from MOG-WT mice, and to a lesser extent in those from MOG-IL6-DIO-KO mice (Fig. 3a and b, bottom). 
A
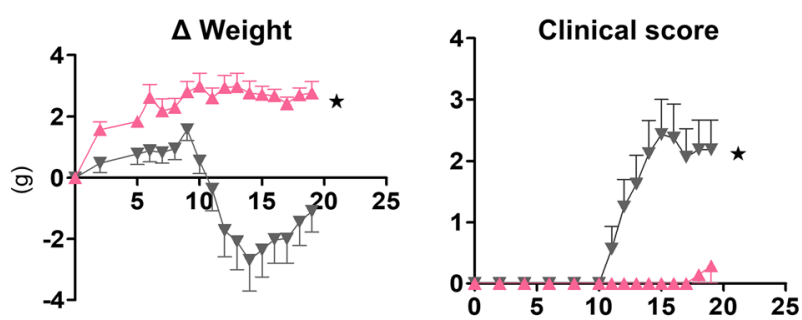

B

Days post-immunization

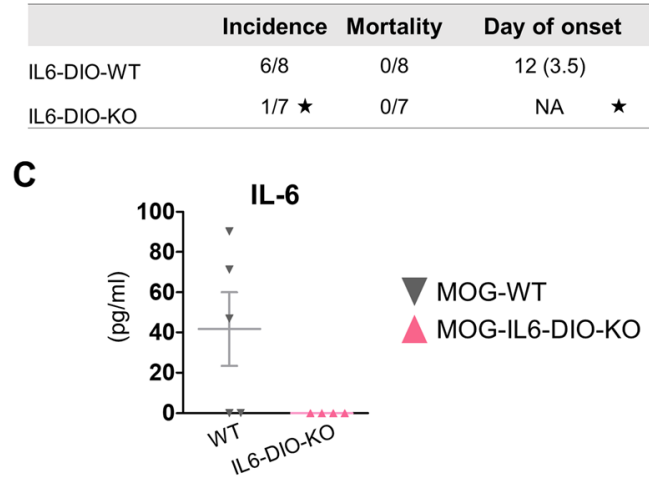

Time to peak

Peak score Median score

$15(3.5) \quad 3(1.6) \quad 3(1.5)$

$N A \quad \star 0(0) \quad \star \quad 0(0) \quad$

D

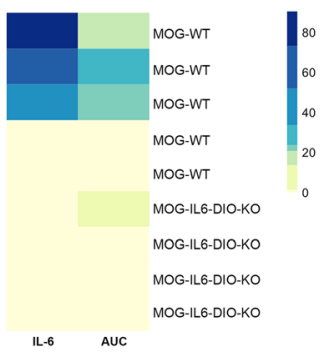

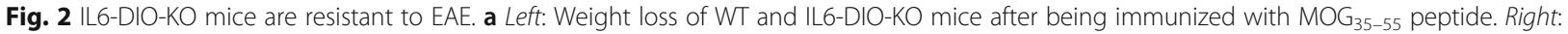
Clinical evaluation of $\mathrm{MOG}_{35-55}$-immunized WT and IL6-DIO-KO mice. IL6-DIO-KO mice did not develop EAE-related symptoms. b Clinical evaluation of EAE. A significant effect on the disease onset, time to peak disease, peak score, and median score between WT and IL6-DIO-KO mice was observed. The incidence, disease onset, and time to peak were calculated using a clinical score $\geq 1$. c Serum IL-6 protein levels were not detected in IL6-DIO-KO mice at 19 dpi. d Heatmap of IL-6 ELISA levels and the respective AUC for each animal in the experiment. Mice with higher disease scores also showed higher IL-6 levels. It is also clear that the two WT mice that did not develop clinical symptoms also did not have increased IL-6 levels. All results are represented as mean $\pm \mathrm{SEM} ; \star p \leq 0.05 \mathrm{vs}$. MOG-WT mice

IL6-DIO-ON ${ }^{\mathrm{Cx} 3 \mathrm{cr} 1}$ mice showed genetic rearrangement of II6 specifically in microglia 1 month after TAM injections We evaluated the ability to reactivate Il6 expression selectively in Cx3cr1-expressing cells and defined the duration of TAM exposure for optimal inversion of exon 2 specifically in Cx3cr1-expressing cells. First, we treated 13 - to 17-week-old mice with TAM for 5 days $(1 \mathrm{mg} /$ day) and 2 weeks afterwards injected them with LPS. We detected IL-6 in the serum of none of LPS-IL6-DIO-KO mice, all of LPS-IL6-DIO-Hz animals, but only in two out of six LPS-IL6-DIO-ON ${ }^{\mathrm{Cx} 3 \mathrm{cr} 1}$ animals, showing that recombination was insufficient (data not shown). Since exon 2 inversion did not work with 5 days of TAM treatment, 10 - to 16 -week-old mice were treated with TAM for 11 days, and with LPS 1 day after the TAM treatment ended. Serum IL-6 now increased modestly in all LPSinjected IL6-DIO-ON ${ }^{\mathrm{Cx} 3 \mathrm{cr} 1}$ mice while all LPS-treated IL6-DIO-KO controls remained undetectable. LPStreated IL6-DIO-Het mice produced high levels of IL-6, as expected (Fig. 4a). This protocol was therefore established for subsequent use.

To test the specificity of recombination in microglial cells, we reactivated $I l 6$ expression in Cx3cr1-expressing cells by treating with TAM for 11 days and, 4 weeks later, extracted DNA from the liver (as a control) and the brain, separating its cells in microglia (CD11b+ sample) and the remainder (CD11b- sample) (Fig. 4b, left). This control is necessary because in $\mathrm{Cx} 3 \mathrm{Cr} 1$-CreER mice, CreER is driven by the $C x 3 c r 1$ promoter, which is expressed in all mononuclear phagocytic cells, which include macrophages [7]. Also, DNA inversion should be present longer in microglia than in Cx3cr1-expressing peripheral cells given microglia's longevity. Therefore, we showed a partial reversion of Il6 in IL6-DIO$\mathrm{ON}^{\mathrm{Cx} 3 \mathrm{cr} 1}$ mice by PCR detection of the reversed band (463 bp) in microglia (CD11b+ sample) but not in a liver sample (where presumably there are circulating Kupffer cells and/or mononuclear cells). As expected, the inverted exon 2 band was observed in both tissues from IL6-DIO-KO mice (Fig. 4b, right).

\section{Reactivation of II6 expression in microglia partially rescues EAE pathology}

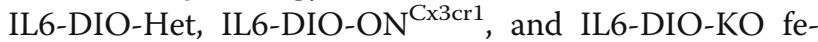
males were used to physiologically confirm Il6 reversion in Cx3cr1-expressing cells. These mice were immunized with $\mathrm{MOG}_{35-55}$ peptide 7 weeks after being injected with a total of $11 \mathrm{mg}$ of TAM. A group of BSA-injected 
A
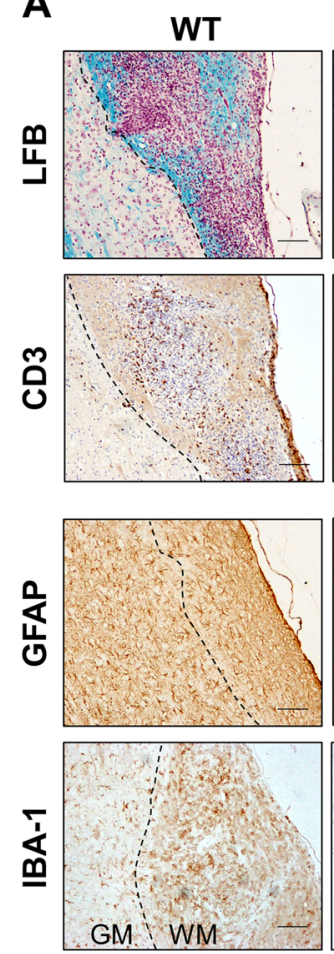

IL6-DIO-KO
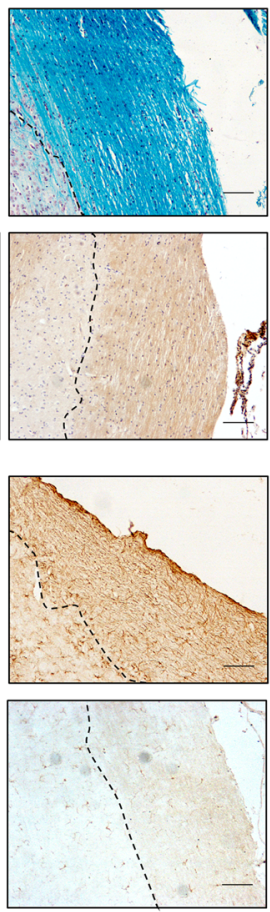

B
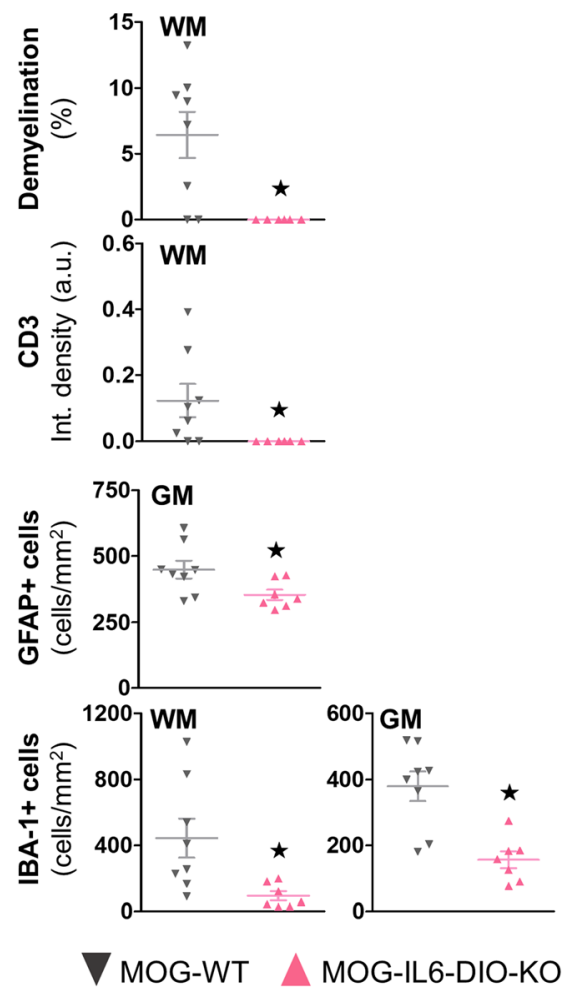

Fig. $3 \mathrm{MOG}_{35-55}$-immunized IL6-DIO-KO mice showed an absence of demyelination and CD3 infiltrates, and limited gliosis. a Representative images of LFB staining and CD3 infiltrates (top), astrogliosis and microgliosis (bottom) of the spinal cord from MOG-WT and MOG-IL6-DIO-KO mice. The contrast of the representative images was enhanced. The lack of IL-6 prevented the infiltration of inflammatory cells. The discontinuous line delimits white matter (WM) from gray matter (GM). Scale bar: $100 \mu \mathrm{m}$. b From top to bottom: Quantification of demyelination by LFB staining and CD3 immunostaining levels (top) and the total number of GFAP+ and IBA-1+ cells (bottom) in the spinal cord from MOG-WT and MOG-IL6DIO-KO mice. All results are represented as mean $\pm \mathrm{SEM} ; \star p \leq 0.05$ vs. MOG-WT mice

female and male IL6-DIO-Het mice (but not injected previously with TAM either LPS) was also included as a control for illustrating the neuropathology of EAE.

Following $\mathrm{MOG}_{35-55}$ immunization, MOG-IL6-DIOHet mice showed the typical ascending paralysis course of EAE, starting at $13 \mathrm{dpi}$ (IQR 1.5) and peaking at 18 dpi (IQR 7) with a clinical score of 2.5 (IQR 0.5) (Fig. 5a, middle and Fig. 5b). Moreover, a prominent body weight loss occurred in these mice in parallel with the clinical signs (Fig. 5a, left). Consistent with our initial results, MOG-IL6-DIO-KO mice were almost completely resistant to EAE (Fig. 5a, b). In contrast, mice with the recovery of $\mathrm{Il6}$ only in microglia (MOG-IL6-DIO-ON ${ }^{\mathrm{Cx} 3 \mathrm{cr1}}$ ) showed a delayed progression of the clinical course, with paralyzed tail at $19 \mathrm{dpi}$ (IQR 10) and peaking at $22 \mathrm{dpi}$ (IQR 7.5), with a peak score of 1 (IQR 1.75) (Fig. 5a and b). As shown in Fig. 5b, the incidence of disease symptoms (clinical score $\geq 1$ ) was $100 \%$ in MOG-IL6-DIOHet and $57 \%$ in MOG-IL6-DIO-ON ${ }^{\text {Cx3cr1 }}$ mice. No mice died during the experiment (27 days) (Fig. 5b).

Significant differences regarding EAE symptomatology were observed between MOG-IL6-DIO-Het and MOG-
IL6-DIO-KO mice, and between MOG-IL6-DIO-Het and MOG-IL6-DIO-ON ${ }^{\mathrm{Cx} 3 \mathrm{cr} 1}$ mice. Furthermore, the severity of the disease was also analyzed by the evaluation of the AUC for each mouse from 21 to $27 \mathrm{dpi}$. We observed differences between MOG-IL6-DIO-Het and MOG-IL6-DIO-ON ${ }^{\mathrm{Cx} 3 \mathrm{cr} 1}$ mice $(p<0.001)$, and MOGIL6-DIO-Het and MOG-IL6-DIO-KO mice $(p<0.001)$. Regarding the day of disease onset, peak score, and median score, MOG-IL6-DIO-Het mice were significantly different from both MOG-IL6-DIO-ON ${ }^{\text {Cx3cr1 }}$ and MOG-IL6-DIO-KO mice (Fig. 5b and Additional file 4). Immunization with BSA did not cause any paralyzing symptoms in IL6-DIO-Het mice. Furthermore, they maintained body weight for all days analyzed, as expected (data not shown).

Il6 mRNA levels were higher in the lower part of the spinal cord (segments T12-Co3) from MOG-IL6-DIOHet than MOG-IL6-DIO-ON ${ }^{\mathrm{Cx} 3 \mathrm{cr} 1}$ mice and were undetectable in MOG-IL6-DIO-KO mice at $27 \mathrm{dpi}$, even though no differences were significant (Fig 6a, top left). However, Il6 levels were in line with the disease extent (AUC) (Fig. 6b). In addition, to dissect the putative role 

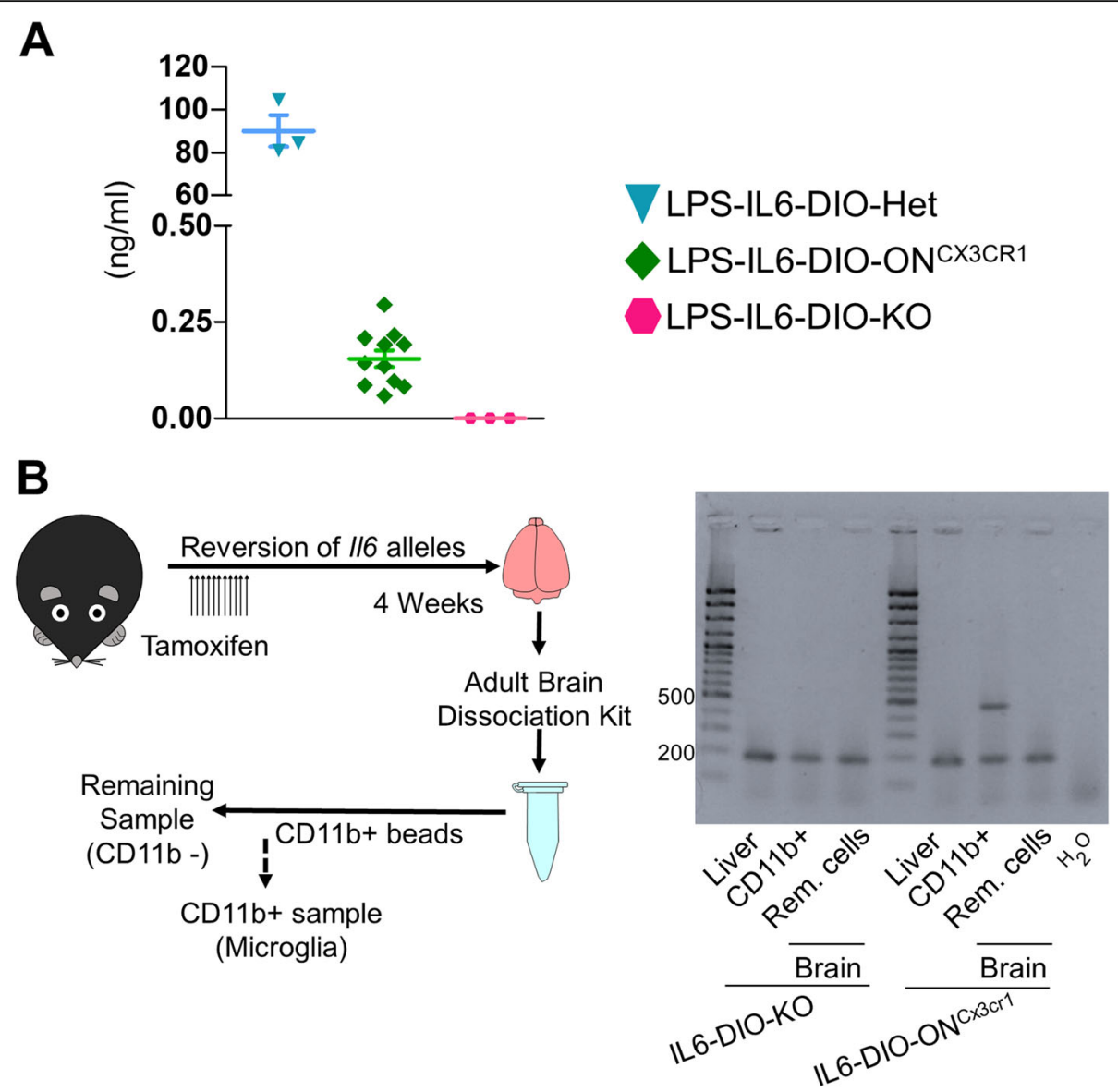

Fig. 4 Reactivation of microglia-derived I/6 expression in IL6-DIO-ON ${ }^{C \times 3 c r 1}$ mice. a Serum IL-6 protein levels of LPS-injected IL6-DIO-Het, IL6-DIO-

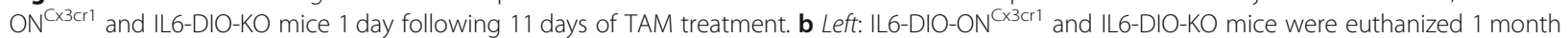
after TAM treatment and the brains were homogenized and incubated with CD11b+ antibody. Besides, the positive cell population was selected using columns with ferromagnetic spheres. Right: The reversion band (463 bp) was only seen in the brain CD11b+ fraction of IL6-DIO-ON ${ }^{C \times 3 c r 1}$ mice. All results are represented as mean \pm SEM

of microglial IL-6 in EAE dynamics, we also analyzed the expression of several genes related to different subsets of T cells (Rorc, which encodes RORyt protein, and Foxp 3 ) and $I l 17 a$ expression. The expression of all these genes was higher in MOG-IL6-DIO-Het mice than MOG-IL6-DIO-KO and MOG-IL6-DIO-ON ${ }^{\text {Cx3cr1 }}$ mice, but no differences were seen between MOG-IL6-DIOKO and MOG-IL6-DIO-ON ${ }^{\text {Cx3cr1 }}$ mice (Fig. 6a).

In accordance with the clinical signs, the upper part of the spinal cord (segments C1-T11) from MOGimmunized IL6-DIO-Het mice, but not those immunized with BSA, showed demyelination of the white matter. Moreover, a significant effect of IL-6 was observed, since MOG-immunized IL6-DIO-KO mice were practically equal to those of BSA-immunized IL6-DIO-Het mice, while recovery of microglial IL-6 in MOG-IL6DIO-ON ${ }^{\mathrm{Cx} 3 \mathrm{cr} 1}$ mice, reverted the demyelination (seemingly partially, but it was not significantly different from MOG-immunized IL6-DIO-Het mice) (Fig. 7).
$\mathrm{MOG}_{35-55}$-immunization also caused an increase of microgliosis (analyzed both by counting IBA-1+ cells and quantitating IBA-1 immunostaining levels) in the white and gray matter of the upper part of the spinal cord (segments C1-T11) from IL6-DIO-Het mice compared with those immunized with BSA. In the white matter, MOG-IL6-DIO-Het showed more microgliosis than MOG-IL6-DIO-KO and MOG-IL6-DIO-ON ${ }^{\text {Cx3cr1 }}$ mice (Fig. 8a and b, and Additional file 5, left top). Although the IBA-1 immunostaining levels in the white matter of MOG-IL6-DIO-ON ${ }^{\mathrm{Cx} 3 \mathrm{cr} 1}$ and MOG-IL6-DIO$\mathrm{KO}$ mice were not different, more IBA-1+ cells were counted in MOG-IL6-DIO-ON ${ }^{\text {Cx3cr1 }}$ than MOG-IL6DIO-KO mice. In the gray matter, MOG-IL6-DIO-Het showed more IBA-1 immunostaining levels than MOGIL6-DIO-KO mice, but not compared with MOG-IL6DIO-ON ${ }^{\text {Cx3cr1 }}$ mice (Fig. 8a, left, and Additional file 5, left bottom). These results were similar to those for demyelination and suggesting that recovery of microglial 
A

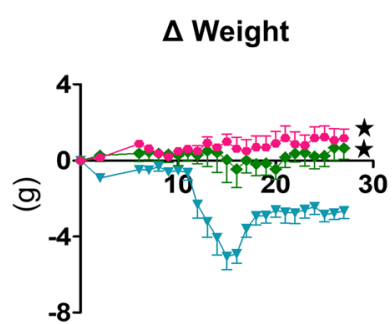

Days post-immunization

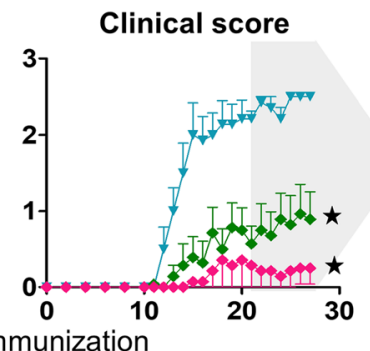

MOG-IL6-DIO-ONCX3cr1

$\nabla$ MOG-IL6-DIO-Het
AUC

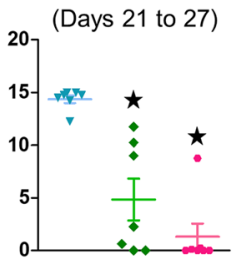

MOG-IL6-DIO-KO

B

\begin{tabular}{lcccccc}
\hline & \multicolumn{2}{c}{ Incidence } & Mortality & Day of onset Time to peak & Peak score & Median score \\
MOG-IL6-DIO-Het & $7 / 7$ & $0 / 7$ & $13(1.5)$ & $18(7.0)$ & $2.5(0.50)$ & $2.5(0.75)$ \\
MOG-IL6-DIO-ONC×3cr1 & $4 / 7$ & $0 / 7$ & $19(10) \star$ & $22(7.5)$ & $1.0(1.75) \star$ & $0.25(1.0) \star$ \\
MOG-IL6-DIO-KO & $1 / 7 \star$ & $0 / 7$ & NA $\star$ & NA $\star$ & $0(0.25) \star$ & $0(0) \star$
\end{tabular}

Fig. 5 IL6-DIO-ON ${ }^{\text {Cx3cr1 }}$ showed mild EAE-related symptoms. a Clinical course of MOG-IL6-DIO-Het, MOG-IL6-DIO-ON ${ }^{\text {Cx3cr1 }}$ and MOG-IL6-DIO-KO

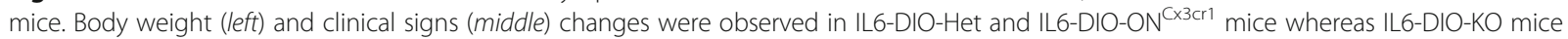
were almost completely resistant to EAE. Area under the curve (AUC; right) was calculated from EAE clinical course for each mouse between days 21 and 27. $\mathbf{b}$ Clinical evaluation of EAE. IL6-DIO-ON ${ }^{\mathrm{CX} 3 \mathrm{Cr} 1}$ and IL6-DIO-KO mice were significantly less affected than IL6-DIO-Het mice. The incidence, disease onset, and time to peak were calculated using a clinical score $\geq 1$. Results in (a) are represented as mean $\pm S E M$, while (b) shows median and IQR (except for incidence and mortality). NA (not applicable) shows the impossibility to calculate the median (given the criterion for disease). $\star p \leq 0.05$ vs. MOG-IL6-DIO-Het mice

IL-6 in MOG-immunized IL6-DIO-ON ${ }^{\text {Cx3cr1 }}$ mice might partially revert the microgliosis in this spinal cord.

A detailed study about the morphology of IBA-1+ cells (microglia/macrophages) in the white matter was also carried out (Fig. 8a, right, and 8b, differentiating three kinds of cells: ramified cells (resting; Fig. 8a, right, panel 1), reactive cells (activated; Fig. 8a, right, panel 2), and round cells (fully activated; Fig. 8a, right, panel 3). The $98.2 \pm$ $0.72 \%$ of IBA- $1+$ cells from negative control (BSAinjected) IL6-DIO-Het mice displayed a ramified morphology. Similar results were seen in MOG-IL6-DIO-KO since $96.7 \pm 0.70 \%$ of IBA- 1 cells displayed a ramified morphology whereas $3.3 \pm 0.7 \%$ seemed to take reactive morphology. Among all cell types, the reactive IBA-1+ cells were the largest population $(78.9 \pm 1.70 \%)$ present in MOG-IL6-DIO-Het mice. The IL6-DIO-ON ${ }^{\text {Cx3cr1 }}$ mice, however, displayed $64.9 \pm 14.7 \%$ and $34.9 \pm 14.5 \%$ of ramified and reactive IBA- $1+$ cells, respectively. A treatmentdependent effect was observed in all three groups of IBA1 + cells. Regarding MOG-immunized mice, MOG-IL6DIO-Het mice showed more ramified and reactive IBA-1+ cells compared with MOG-IL6-DIO-KO and IL6-DIO$\mathrm{ON}^{\mathrm{Cx} 3 \mathrm{cr} 1}$ mice, and IL6-DIO-ON ${ }^{\mathrm{Cx} 3 \mathrm{cr} 1}$ mice showed more reactive, but not ramified IBA-1+ cells compared with MOG-IL6-DIO-KO mice. Concerning round IBA-1+ cells, a significant increase was appreciated in MOG-IL6-DIOHet compared with MOG-IL6-DIO-KO mice whereas a trend $(p=0.098)$ was shown in MOG-IL6-DIO-Het as to IL6-DIO-ON ${ }^{\text {Cx3cr1 }}$ mice. The same trend was observed among MOG-IL6-DIO-KO and IL6-DIO-ON ${ }^{\mathrm{Cx} 3 \mathrm{cr} 1}$ mice $(p=0.098)$.

In addition, we analyzed the astrogliosis by counting GFAP+ cells and studying GFAP immunostaining levels in the upper part of the spinal cord (segments C1-T11). In particular, no differences between treatments and genotypes were appreciated concerning GFAP immunostaining levels in the white matter, perhaps because of the myelin tracts also seemed to be immunostained, thus increasing background levels (Fig. 8a, middle and Additional file 5, right top). Regarding the gray matter, $\mathrm{MOG}_{35-55}$ immunization showed a trend to increase the GFAP immunostaining levels in IL6-DIO-Het compared with those with BSA immunization $(p=0.067)$, being this difference statistically significant when we counted the number of GFAP+ cells. Both GFAP immunostaining levels and the number of GFAP+ cells were different among MOG-IL6-DIO-Het and MOG-IL6-DIO-KO mice (Fig. 8c and Additional file 5, right bottom). Although GFAP immunostaining levels in the gray matter were not statistically significant between MOG-IL6-DIO-Het and MOG-IL6-DIO-ON ${ }^{\mathrm{Cx} 3 \mathrm{cr} 1}$ mice, we counted a higher number of GFAP+ cells in MOG-IL6-DIO-Het than MOG-IL6-DIO-ON ${ }^{\text {Cx3cr1 }}$ mice in this matter (Fig. 8c and Additional file 5, right bottom).

We also classified the number of astrocytes in the gray matter of the spinal cord according to their ramified 


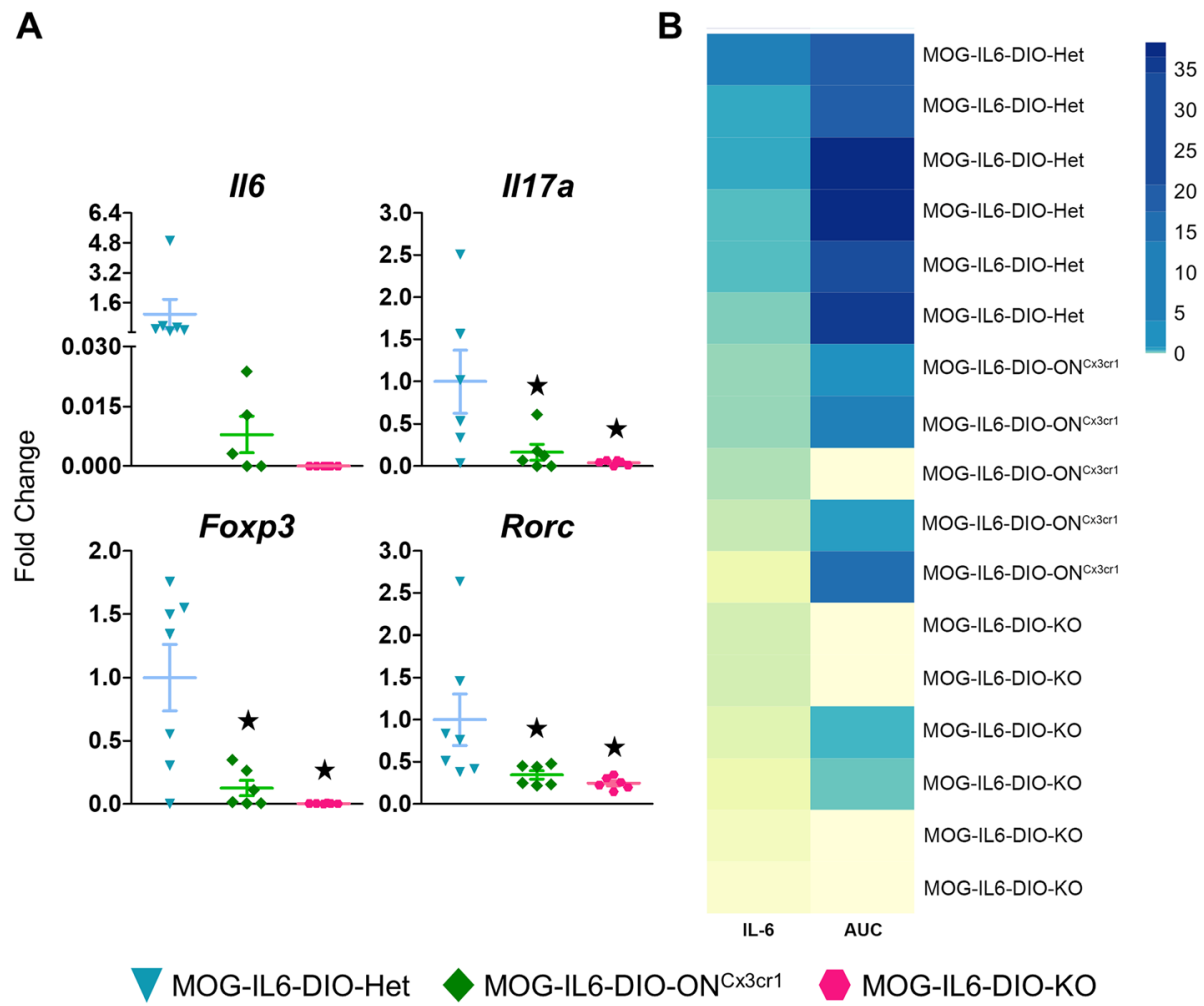

Fig. 6 Inflammation-related genes are down-regulated accordingly to disease severity. The changes of inflammation-related genes expression were studied using the upper part of the spinal cord (segments C1-T11) of three groups of mice euthanized at $27 \mathrm{dpi}$. a Gene expression of $/ 16$, $1 / 17 a$, Foxp3, and Rorc was lower in MOG-IL6-DIO-ON ${ }^{\text {Cx3cr1 }}$ and MOG-IL6-DIO-KO mice than in MOG-IL6-DIO-Het control mice. b Heatmap of I/6 expression and AUC of disease score. 116 was only increased in mice with higher AUC. Results in (a) are represented as mean \pm SEM; $\star p \leq 0.05$ vs. MOG-IL6-DIO-Het mice

(Fig. 8a, right, panel 4) or reactive (Fig. 8a, right, panel 5) morphology. In IL6-DIO-Het mice immunized with BSA, $93.5 \pm 1.15 \%$ of GFAP+ cells displayed a resting ramified morphology, while in those immunized with $\mathrm{MOG}_{35-55}, 33.9 \pm 4.76 \%$ had a ramified morphology and $66.1 \pm 4.76 \%$ GFAP+ cells seemed to take a starshaped morphology. In contrast, $85.0 \pm 2.93 \%$ and 63.2 $\pm 10.30 \%$ of GFAP+ cells displayed a resting morphology in the gray matter of MOG-IL6-DIO-KO and IL6-DIO$\mathrm{ON}^{\mathrm{Cx} 3 \mathrm{cr} 1}$ mice, respectively. $\mathrm{MOG}_{35-55}$-immunization decreased ramified GFAP+ cells, but increased reactive GFAP+ cells in IL6-DIO-Het mice compared with those with BSA-immunization. The number of ramified GFAP+ cells were lower in MOG-IL6-DIO-Het and MOG-IL6-DIO-ON ${ }^{\mathrm{Cx} 3 \mathrm{cr} 1}$ than MOG-IL6-DIO-KO mice, and a trend to increase the number of ramified GFAP+ cells was also appreciated in MOG-IL6-DIO-ON ${ }^{\text {Cx3cr1 }}$ compared to MOG-IL6-DIO-Het mice $(p=0.081)$. Genotypic differences were also observed regarding the number of GFAP+ cells with a reactive morphology, which was higher in MOG-IL6-DIO-Het than MOGIL6-DIO-KO and MOG-IL6-DIO-ON ${ }^{\text {Cx3cr1 }}$ mice. The recovery of microglial IL-6 in MOG-IL6-DIO-ON ${ }^{\text {Cx3cr1 }}$ mice tended to increase the number of reactive GFAP+ cells in comparison with MOG-IL6-DIO-KO mice ( $p=$ 0.063; Fig. 8b, right).

\section{Discussion}

Traditional methods for creating reversible $\mathrm{KO}$ mice are based on strategies that require numerous crossings or the continuous administration of drugs. The main methods are (1) cassette stop/trap flanked by loxP sites [33-35], (2) Tet-off and tet-on [36-38], and (3) LOFT: LoxP-flippase (FLP) recognition target (FRT) Trap [39].

The stop-cassette strategy is based on creating a knockout by introducing transcription termination elements that prevent the complete production of the target protein. Reversibility is achieved by driving Cre expression with tissue-specific promoters fused with the estrogen receptor (ER) paired with tamoxifen injection for temporal and spatial specificity. Alternatively, injection of a Cre-expressing virus can rescue in a similarly time- and spatial-specific manner. However, stop cassettes can be leaky and trace expression must be assessed empirically. 


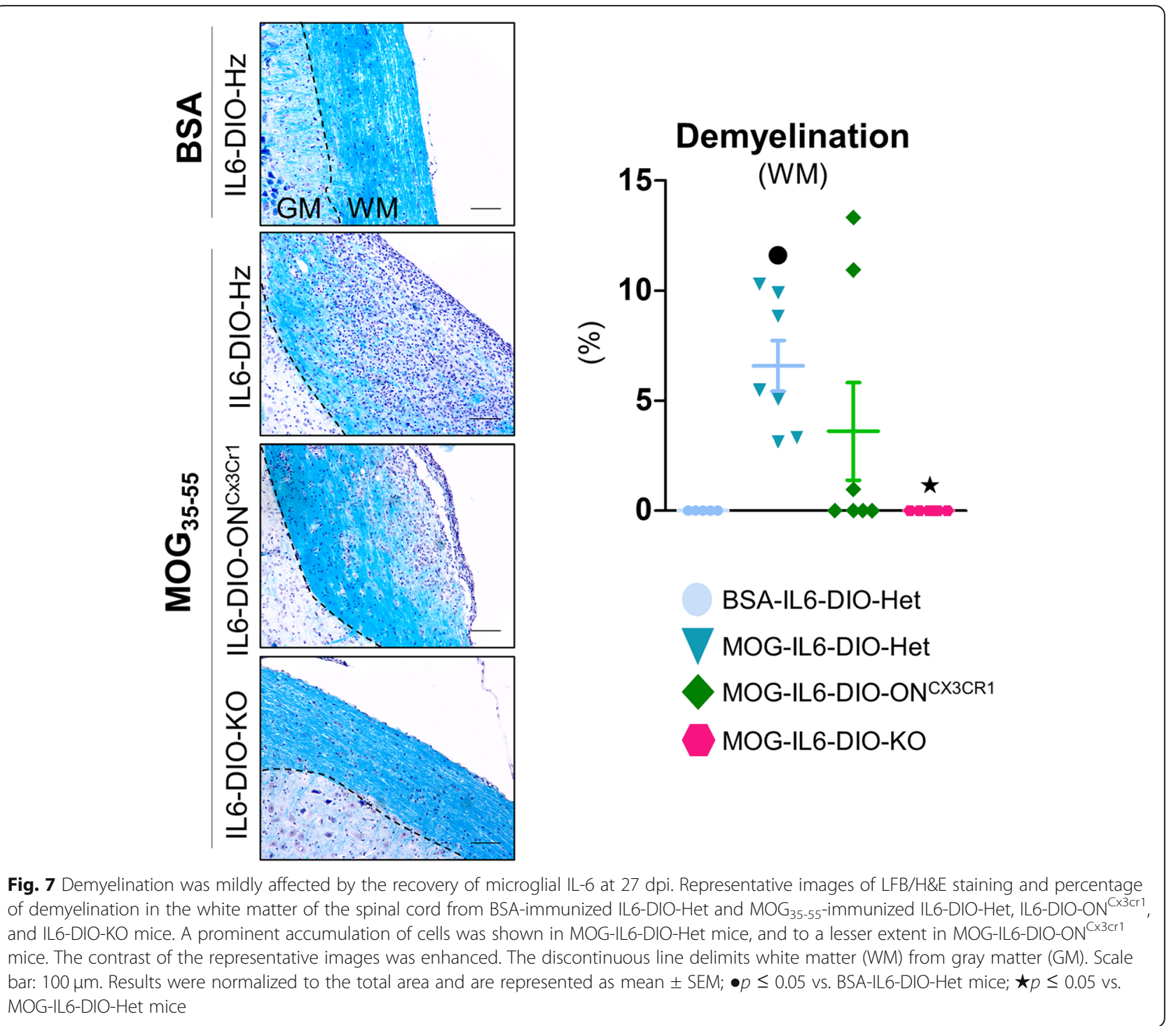

Tet-off and Tet-on use Escherichia coli's tetO operon to induce expression of the adjacent gene. In the "off" system, the mouse becomes a $\mathrm{KO}$ upon (and only during) administration of tetracycline (or, in practice, its derivative doxycycline), which then binds to a tetracyclinecontrolled transactivator (tTA) that has been engineered to be constitutively expressed, and prevents the transcription of the gene downstream of tetO. In the "on" system, the transactivator is modified to have the reverse phenotype (rtTA), i.e., it only binds to tetO in the presence of doxycycline. In this case, the animal is initially a $\mathrm{KO}$, and it will only produce the target protein upon drug administration. As downsides, both strategies depend on continuous administration of the drug to maintain the animal's genotype; the use of a stop cassette with loxP sites in order to obtain tissue specificity has the problem mentioned above; and furthermore, they require extensive breeding and animals that are transgenic for multiple genes.

The LOFT system uses two modified alleles for the gene of interest, one of them floxed and the other with an frt-flanked gene-trap cassette (that also has Neo and GFP) inserted in an intron. The latter will produce by default an inactive fusion protein with the $\mathrm{N}$-terminal domain of the target protein and Neo. When Cre is expressed, the animal will become a $\mathrm{KO}$. If there is additionally flippase activity, the phenotype will be reversed. This method requires a considerable number of crossings to incorporate the two alleles and recombinases making it labor- and cost-intensive. In addition, given that the allele with the gene-trap is null by default, the gene must be haplosufficient.

To overcome these pitfalls, we have generated a conditional and reversible $\mathrm{KO}$ mouse using the widely used in 


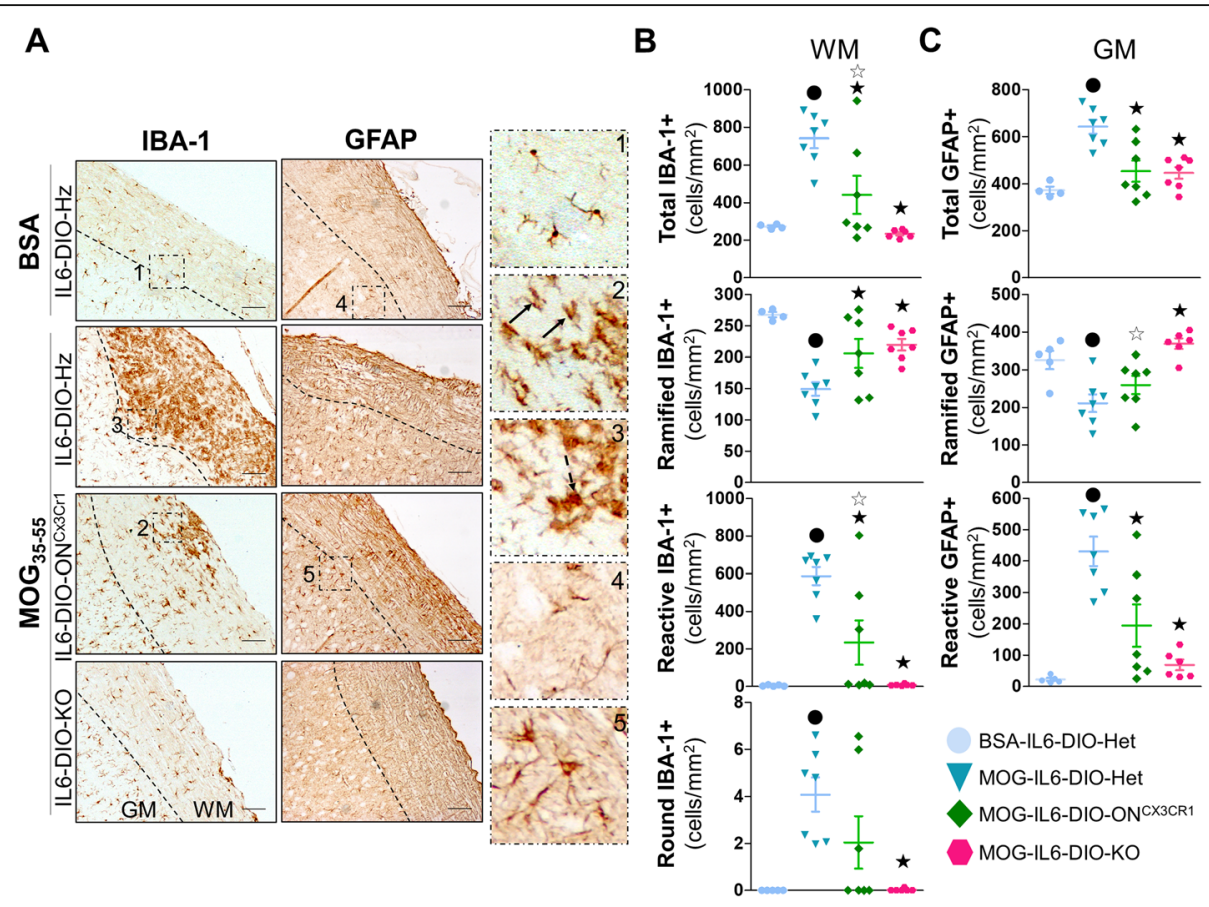

Fig. 8 Microglial IL-6 plays a minor role in the regulation of microgliosis and astrogliosis. a Representative images of IBA-1 (left) and GFAP (middle) immunostaining of BSA-immunized IL6-DIO-Het and MOG $35-5$-immunized IL6-DIO-Het, IL6-DIO-ON ${ }^{C \times 3 c r 1}$ and IL6-DIO-KO mice. High magnification (right) of (a) showing the different morphologies of IBA-1+ and GFAP+ cells: (1) ramified, resting IBA-1+ cells; (2) reactive IBA-1+ cells, with shorter and thicker cell processes (black arrows); (3) round IBA-1+ cells (dashed black arrow); (4) Ramified, resting GFAP+ cells; (5) reactive, hypertrophic GFAP+ cells. The contrast of the representative images was enhanced. The discontinuous line delimits white matter (WM) from gray matter (GM). Scale bar: $100 \mu \mathrm{m}$. b The total number of IBA-1+ cells and the number of IBA-1+ cells showing a resting phenotype (ramified), partially activated (reactive), and fully activated (round) counted in the white matter of the spinal cord from BSA-immunized WT and MOG 35 -55-immunized IL6-DIOHet, IL6-DIO-ON ${ }^{\text {C3Cr1 }}$, and IL6-DIO-KO mice. c The total number of GFAP+ cells and the number of GFAP+ cells showing a resting phenotype (ramified) and activated (reactive) counted in the gray matter of the spinal cord from BSA-immunized WT and MOG ${ }_{35-55}$-immunized IL6-DIO-Het, IL6-DIO-ON ${ }^{\mathrm{C} 3 \mathrm{Cr} 1}$, and IL6-DIO-KO mice. All results were relativized per total area and are represented as mean \pm SEM; $\bullet p \leq 0.05$ vs. BSA-IL6-DIOHet mice; $\star p \leq 0.05$ vs. IL6-DIO-Het mice. $\iota_{3} p \leq 0.05$ vs. IL6-DIO-KO mice

opto- and chemogenetics double-inverted, open-readingframe paradigm $[4,29-31]$. This technique consists of flanking exons of interest by pairs of incompatible loxP sites such that Cre recombinase flips the exons and then locks them into the flipped orientation [4, 29-31]. Exon 2 of the $I l 6$ gene was inverted in IL6-DIO-KO mice causing a depletion of IL-6 protein (undetectable after inflammation challenges such as LPS and EAE), which is equivalent to results with the total IL-6 KO mice [12, 40]. In our EAE paradigm, almost all IL6-DIO-KO mice were resistant to $M_{35} G_{35-55}$-inducible EAE, except for one, which presented EAE-related symptoms even without showing serum IL-6 levels, as previously observed with other IL-6 KO models [11, 12, 41].

The EAE-related symptoms of IL6-DIO-KO mice were similar to those of WT mice immunized with $\mathrm{MOG}_{35-55}$ peptide and administered intraperitoneally with anti-IL6 receptor monoclonal antibody (clone MR16-1) at the same day of EAE induction, but different to those of WT mice immunized with $\mathrm{MOG}_{35-55}$ peptide and treated with clone MR16-1 after disease onset [42]. In addition, WT mice immunized with $\mathrm{MOG}_{35-55}$ peptide and orally treated during ongoing EAE with tocilizumab, a humanized monoclonal antibody that inhibits IL-6Rmediated signaling, showed a drastic reduction of EAErelated symptoms [43]. It is therefore clear that IL-6 signaling is involved in the pathogenesis of EAE. However, given the functional complexity of IL-6, the priority of many groups during the last decades has been to study the multiple cellular sources of IL- 6 to better understand the contribution of each one to the EAE pathogenesis. Until now, the roles of IL-6 in a specific context have been described through the use of mice lacking IL-6 throughout the body or in specific cells (total and conditional IL-6 KO mice, respectively). Nevertheless, the interpretation of the results using the latter model is difficult because the data could be the result of a compensatory response from IL- 6 of other cells. With our new conditional reversible IL-6 KO mouse, we now have a tool that allows for the reactivation of Il6 expression in cells expressing Cre recombinase. Thus, we were able to recover microglial IL- 6 in the reversible 
total IL-6 KO mouse through breeding to $\mathrm{C} x 3 \mathrm{cr} 1$-CreER mice and subsequent TAM administration. Our results suggest that recovery of microglial IL- 6 gives rise to a mild version of EAE, albeit with a limited role in the regulation of the inflammatory cascade. In conclusion, we have verified that the new reversible total IL-6 KO is an excellent tool to study the role of specific cellular sources of IL-6 within a recovery-of-function paradigm.

Although EAE is considered a peripheral disease, microglia, besides CD4+ cells, have been described as responsible for EAE initiation [23, 44]. In fact, in $\mathrm{MOG}_{35-55}$-immunized C57BL/6 mice, CD4+ $\mathrm{T}$ cells infiltrated the brain before mice developed EAErelated symptoms, coinciding with activation of $\mathrm{CD} 11 \mathrm{~b}+$ microglia and production of IL- $1 \beta, \mathrm{TNF}-\alpha$, and IL-6 [45]. Furthermore, we have recently observed that microglia are one of the main brain sources of IL-6 during EAE pathogenesis and microglial Il6-deficient females seemed to be less affected by $\mathrm{MOG}_{35-55}$ immunization than their controls [19]. In this paper, we have focused on studying the effect of selective recovery of microglial IL- 6 expression by crossing IL6-DIO-KO with Cx3cr1-CreER mice to show the applicability of this new system and to gain insight into the putative role(s) of microglial IL-6 in the EAE disease.

In $\mathrm{Cx} 3 \mathrm{Cr} 1 \mathrm{CreER}$ mice, Cre is driven by the $C x 3 \mathrm{cr} 1$ promoter, which is broadly expressed in the mononuclear phagocytic cells and it is only expressed in microglia in the adult brain $[7,46,47]$. Furthermore, Cre recombinase protein of these mice is fused to the estrogen ligand-binding domain (CreER), thereby requiring TAM for its activation [6, 7]. Then, IL6-DIO$\mathrm{ON}^{\mathrm{Cx} 3 \mathrm{cr} 1}$ mice should revert $I l 6$ expression in Cx3cr1-expressing cells, including macrophages and microglia, after TAM treatment. Since IL-6 protein levels in serum are practically undetected in basal conditions, mice were also injected with LPS following TAM treatment to increase IL-6 production. The recovery of IL-6 occurred in IL6-DIO-ON ${ }^{\mathrm{Cx} 3 \mathrm{cr} 1}$ mice administrated with TAM for 11 days and not for 5 days. However, IL-6 production was lower in LPSIL6-DIO-ON $^{\text {Cx3cr1 }}$ than LPS-IL6-DIO-Het mice, probably due to the fact that only CX3CR1+ cells in IL6-DIO-ON ${ }^{\mathrm{Cx} 3 \mathrm{cr} 1}$ mice would be responsible for IL-6 production following LPS administration while all cells would produce IL-6 protein in IL6-DIO-Het mice (these mice have one WT allele and one DIO allele; in addition, TAM administration would cause the Cre activation and subsequent $I l 6$ reversion of the DIO allele in CX3CR1+ cells in these mice). We did not attempt longer TAM administrations given that our current dose is already at the higher end of the reported spectrum $[48,49]$ and the drug is a known immunosuppressant which could interfere with EAE evaluation [50].

The longevity and limited self-renewal are the main features of microglia which distinguishes them from other mononuclear phagocytic cells [51, 52]. In fact, Goldmann and colleagues observed that inducible Cx3cr1CreER:R26-YFP mice, obtained by crossing Cx3Cr1CreER mice with R26-YFP mice, displayed YFPlabeled microglia at all times after TAM treatment whereas other mononuclear phagocytic cells lost the YFP-label for the first 4 weeks after treatment [6]. We also observed similar results using our inducible microglial IL-6 KO mice, obtained by crossing Cx3Cr1CreER mice with $I l 6^{\text {lox/lox }}$ mice, since microglia of these mice, but not other brain cells or liver (assuming this tissue as representative of macrophages/monocytes), kept the genomic modification 4 weeks following TAM administration [26]. Therefore, we also performed the validation of IL6-DIO-ON ${ }^{\mathrm{Cx} 3 \mathrm{crl}}$ mice at the same time as above mentioned. As expected, IL6-DIO-ON ${ }^{\text {Cx3cr1 }}$ mice kept the reversion of the DIO allele in microglia, but not in other brain cells or liver, 4 weeks after being treated with TAM for 11 days. These data confirm that the contribution of mononuclear phagocytic cells to results obtained with IL6-DIO-ON ${ }^{\text {Cx3cr1 }}$ mice from 4 weeks following TAM-treatment will be limited.

Reactivation of microglial $\mathrm{Il} 6$ expression in $\mathrm{MOG}_{35-55^{-}}$ immunized IL6-DIO-ON ${ }^{\mathrm{Cx} 3 \mathrm{cr} 1}$ females developed some of the prototypical paralyzing symptoms showing that despite the fact that EAE is considered essentially a peripheral disease, microglial $I l 6$ was sufficient to trigger a mild version of EAE-related symptoms. Results are in line with females lacking IL-6 in microglia, which tended to reduce EAE score during the acute phase of the disease in comparison with their controls [19]. Clinical signs of IL6-DIO-ON ${ }^{\mathrm{Cx} 3 \mathrm{cr} 1}$ mice were lower than those of IL6-DIO-Het animals, reinforcing the idea that IL-6 derived from other cellular sources is also involved in the EAE-related symptomatology. Of note, scores (AUC) within the IL6-DIO-ON ${ }^{\mathrm{Cx} 3 \mathrm{cr} 1}$ group have a bimodal distribution, which also does not fully correlate with $I l 6$ expression. This could be due to only partial recovery of microglial $I l 6$ or to this source not being as relevant to disease progression and just indicating a stochastic process. Indeed, T-cell- and astrocyte-derived Il6-deficient mice showed ameliorated symptoms compared with their controls after being immunized with $\mathrm{MOG}_{35-55}$ [17-19]. However, deficiency of dendritic cell-derived $I l 6$ caused that naïve $\mathrm{T}$ cells could not differentiate into Th17 cells blocking EAE score in mice [17]. In contrast, Il6 deficiency in other cells caused no (in neurons), contradictory (in B cells), or aggravated (macrophage) effects in the regulation of EAE-related symptoms $[16,17,19]$. 
The expression of mRNA encoding Il6 was higher in the lower part of the spinal cord (segments T12-Co3) from MOG-IL6-DIO-Het than MOG-IL6-DIO-ON ${ }^{\text {Cx3cr1 }}$ mice and it was absent in MOG-IL6-DIO-KO mice at 27 dpi, but high variability precluded any statistically significant effect between genotypes. Moreover, we cannot rule out that differences would exist at other time points. EAE is a complex disease in which IL-6 undoubtedly plays an important role since it suppresses the differentiation of TGF- $\beta$-induced Foxp $3+$ Treg, and with TGF- $\beta$ promotes the differentiation of pathogenic TH17 cells from naive $\mathrm{T}$ cells [15]. In this paper, IL6-DIO-KO mice showed downregulation of Rorc expression, the crucial transcription factor of Th17, suggesting a reduction of Th17 differentiation, and a similar trend regarding Il17 mRNA levels in the spinal cord compared to IL6-DIO-Het. Indeed, less percentage of Th17 cells infiltrating the CNS was previously reported using

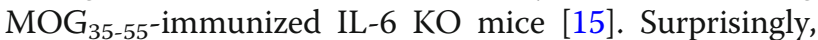
the recovery of microglial IL-6 did not modify $I l 17$ expression in comparison with the lack of total IL-6 in mice at $27 \mathrm{dpi}$, as it was observed in IL-17 immunostaining levels in microglial Il6 deficient mice at 27-29 dpi [19]. Besides, the expression of mRNA encoding Foxp3, the master transcription factor of Treg, was also downregulated by lack of total IL-6, in agreement with IL-6 KO mice, whose cerebellum showed less percentage of Foxp3 cells per infiltrate than those of MOG $35-55$-immunized WT mice [12]. Interestingly, another work reported that IL-6 KO mice (crossed previously with the Foxp3gfp.KI mice to monitor Treg cells) showed more Foxp3+ Treg cells in the draining lymph nodes than their controls [53], which suggests the necessity of further studies to determine these discrepancies. Foxp 3 and Rorc expression levels were practically equal among MOG-IL6-DIO-ON ${ }^{\mathrm{Cx} 3 \mathrm{cr} 1}$ and MOG-IL6-DIO-KO mice at $27 \mathrm{dpi}$, but distinct to MOG-IL6-DIO-Het, suggesting that other cellular sources of IL6, and not microglia, might regulate these transcription factors at this time point. IL-6 has also been described as a regulatory protein of some inflammation-related genes at 15-20 dpi $[12,54]$. In particular, microglial Il6 deficiency caused changes in Gfap and Cd206 (alternatively activated macrophages/microglia (M2) phenotypic marker) expression in the lower part of the spinal cord (segments T12-Co3) from mice at $15 \mathrm{dpi}$ [19]. Future studies are needed to determine what occurs regarding inflammation-related markers in the MOG-IL6-DIO-ON ${ }^{\mathrm{Cx} 3 \mathrm{cr} 1}$ mice at peak disease.

The histological analysis of the upper part of the spinal cord (segments C1-T11) from MOG-IL6-DIO$\mathrm{KO}$ mice revealed a decrease of demyelination and limited invasion of $\mathrm{CD} 3+$ in the white matter, as previously observed using other IL-6 KO models immunized with $\mathrm{MOG}_{35-55}[11,12]$, and highlighting once again a critical role of IL-6 in the prototypical autoimmune disorder. Interestingly, the recovery of microglial IL-6 in MOG-IL6-DIO-ON ${ }^{\text {Cx3cr1 }}$ females seemed to revert partially the demyelination, in agreement with results obtained with microglial Il6-deficient females at $29 \mathrm{dpi}$, which showed a trend to decrease the demyelination [19], pointing to in both cases that IL-6 derived from microglia might regulate the demyelination. As stated, another key pathological feature of EAE is the inflammatory response of astrocytes and microglia/macrophages, therefore we quantified the GFAP and IBA-1 immunostaining levels and classify IBA-1 and GFAP+ cells according to their morphology. In general, the lack of IL- 6 reduced the microgliosis and astrogliosis in the spinal cord of mice, displaying most of IBA- $1+$ and GFAP+ cells a resting morphology, in line with IL-6 KO [12]. The recovery of microglial IL-6, in agreement with the mild clinical signs of MOG-IL6-DIO-ON ${ }^{\mathrm{Cx} 3 \mathrm{cr} 1}$ mice, increased the number of reactive IBA-1+ cells in the white matter and a similar trend was observed regarding IBA-1 immunostaining levels in the gray matter. Somewhat surprisingly, considering the relevance of IL-6 in inflammatory processes of EAE, is that the recovery of microglial IL-6 in MOG-IL6-DIO-ON ${ }^{\text {Cx3cr1 }}$ mice did not modify the total number of GFAP+ cells in the gray matter, although it reduced the ramified GFAP+ cells and tended to increase the reactive GFAP+ cells. Taking into account the results obtained with microglial Il6-deficient females at $29 \mathrm{dpi}$, in which deficiency of microglial $I l 6$ tended to reduce IBA-1 immunostaining levels in the white matter, but it did not play a relevant role in the regulation and GFAP immunostaining levels [19], it seems that microglial Il6 may regulate the microgliosis, but it would have a limited function in the regulation of astrogliosis during the inflammatory response to EAE.

\section{Conclusions}

In summary, we have presented and validated a novel reversible total IL-6 KO mouse, whose Il6 expression was recovered specifically in microglia. The recovery of microglial Il6 was sufficient to develop a mild version of EAE-related symptoms in females, although it seemed to have a minor role in the regulation of the inflammatory cascade. Furthermore, the reversion of Il6 expression in different cells using this IL6-DIO-KO mouse model, combined with cell-specific Cre transgenic lines, would be key to elucidate the specific functions of IL-6 from each cellular source and study cell-specific IL- 6 targeting strategies in EAE and a wide range of autoimmune and neuroinflammatory diseases. Indeed, our group is currently analyzing the role of IL- 6 in a mouse model of 


\begin{abstract}
Alzheimer's disease and in a mouse model of progressive encephalopathy resembling Leigh syndrome using this novel strategy. Furthermore, the IL6-DIO-KO mouse model will be available for the scientific community and will be distributed worldwide following material transfer agreements with Universitat Autònoma de Barcelona. Finally, we also encourage other investigators to take up this reversible and conditional knock-out strategy to carry out rescue experiments in other genetic models.
\end{abstract}

\section{Supplementary information}

Supplementary information accompanies this paper at https://doi.org/10. 1186/s12974-020-01969-0.

Additional file 1. Probe sequence. Primers FW:5'-CAGCATCTCATCTGAG TTCCG-3' and Rv:5'-CTCACTGTTCACAAAGCACAGG-3' were used to design a unique probe of $621 \mathrm{bp}$.

Additional file 2. DNA sequencing of the modified //6 gene of the IL6DIO-KO mice. The resulting sequence had $1361 \mathrm{bp}$ and the localization, size and color code of the genetic structures (exons and protein binding zones) detected in this sequence are explained in the figure legend. The loxP and lox2272 cassettes (purple and green, respectively) are in the opposite orientation, then sequence between them (including the exon 2) will be reverted after Cre action.

Additional file 3. Kaplan-Meier analysis in the EAE experiment with IL6DIO-KO and WT mice. Mice were assigned status "sick" on the first day their score was $\geq 1$ and the proportion of sick mice was represented for each day. Mice that never showed scores above the threshold were censored. The time-course of disease for IL6-DIO-KO mice is significantly delayed, with only one mouse fulfilling the disease criterion (no median). Table shows number of mice, sick mice, median day and 95\% confidence interval. NA (not applicable) indicates impossibility to calculate value.

Additional file 4. Kaplan-Meier analysis in the EAE experiment with IL6DIO-ON ${ }^{\text {CX3cr1 }}$, IL6-DIO-KO and IL6-DIO-Het mice. Mice were assigned status "sick" on the first day their score was $\geq 1$ and the proportion of sick mice was represented for each day. The time-course of disease for IL6$\mathrm{DIO}-\mathrm{ON}^{\mathrm{CX} \mathrm{Cr}^{1}}$ and IL6-DIO-KO mice is significantly delayed. IL6-DIO-KO again showed almost complete resistance to $E A E$, with only one mouse actually developing the disease (no median). Tables show number of mice, sick mice, median day and 95\% confidence interval; as well as post hoc pair-wise comparisons. NA (not applicable) indicates impossibility to calculate value.

Additional file 5. Quantification of IBA-1 and GFAP immunostaining levels shown in Fig. 8. Quantification was carried out in both white and gray matter of BSA-immunized IL6-DIO-Het and MOG $35-55^{-i m m u n i z e d ~ I L 6-~}$ $\mathrm{DIO}-\mathrm{Het}, \mathrm{IL} 6-\mathrm{DIO}-\mathrm{ON}{ }^{\mathrm{C} \times 3 \mathrm{Cr} 1}$ and IL6-DIO-KO mice. All results were relativized per total area and are represented as mean \pm SEM; $\bullet p \leq 0.05$ vs. BSA-IL6-DIO-Het mice; $\star p \leq 0.05$ vs. IL6-DIO-Het mice.

\section{Abbreviations}

AUC: Area under the curve; BSA: Bovine serum albumin; CD11b: Cluster of differentiation molecule 11b; CNS: Central nervous system; DIO: Doubleinverted open reading frame; EAE: Experimental autoimmune encephalomyelitis; GFAP: Glial fibrillary acidic protein; GM: Gray matter; IBA1: Ionized calcium-binding adaptor molecule-1; IL-6: Interleukin-6; LFB: Luxo Fast Blue; LOFT: LoxP-flippase (FLP) recognition target (FRT) Trap; LPS: Lipopolysaccharide; MOG $_{35-55}$ : Myelin oligodendrocyte glycoprotein 3555 peptide; MS: Multiple sclerosis; TAM: Tamoxifen; WM: White matter; YFP: Yellow fluorescent protein

\section{Acknowledgements}

We thank Profs. Marco Prinz and Steffan Jung for providing Cx3cr1-CreER mice.

\section{Authors' contributions}

Conceptualization: JH and RDP; methodology: PS, OFG, GC, KA, AE, MG; formal analysis and investigation: PS, OFG and JH; writing - - original draft preparation: PS and OFG; writing - - review and editing: JH and RDP; funding acquisition: $\mathrm{JH}$. All authors read and approved the final manuscript.

\section{Funding}

PS, OFG, KA, and AE, acknowledge the support of BES-2015-071959, FPU2012-00365, FPU17/02065 and La Marató de TV3 20142210, fellowships respectively. This study was supported by Ministerio de Economía y Competitividad y Fondo Europeo de Desarrollo Regional SAF2014-56546-R and RTI2018-101105-B-I00 to J.H.

\section{Availability of data and materials}

The datasets used and/or analysed during the current study are available from the corresponding author on reasonable request.

\section{Ethics approval and consent to participate}

All experiments were approved by the Ethics Committee on Animal Experiments of the Universitat Autònoma de Barcelona and the Generalitat de Catalunya (Refs. 3782 and 9684, respectively).

\section{Consent for publication}

Not applicable.

\section{Competing interests}

Not applicable.

\section{Author details}

${ }^{1}$ Institute of Neurosciences and Department of Cellular Biology, Physiology and Immunology, Animal Physiology Unit, Faculty of Biosciences, Universitat Autònoma de Barcelona, 08193 Barcelona, Spain. ${ }^{2}$ Current affiliation: Department of Pediatrics, Division of Molecular Genetics, Columbia University Irving Medical Center, New York 10032, USA. ${ }^{3}$ Department of Biochemistry, Genome Sciences, and Howard Hughes Medical Institute, University of Washington, Seattle, WA 98195, USA.

Received: 22 July 2020 Accepted: 28 September 2020

Published online: 15 October 2020

\section{References}

1. Palmiter RD, Brinster R. Transgenic mice. Cell. 1985;41:343-5.

2. Sternberg N, Hamilton D. Bacteriophage P1 site-specific recombination I. recombination between loxP sites. J Mol Biol. 1981:150:467-86.

3. Metzger D, Clifford J, Chiba H, Chambon P. Conditional site-specific recombination in mammalian cells using a ligand-dependent chimeric Cre recombinase. Proc Natl Acad Sci U S A. 1995;92:6991-5.

4. Atasoy D, Aponte $Y$, Su HH, Sternson SM. A FLEX switch targets Channelrhodopsin-2 to multiple cell types for imaging and long-range circuit mapping. J Neurosci. 2008:28:7025-30.

5. Quintana A, Erta M, Ferrer B, Comes G, Giralt M, Hidalgo J. Astrocyte-specific deficiency of interleukin-6 and its receptor reveal specific roles in survival, body weight and behavior. Brain Behav Immun. 2013;27:162-73.

6. Goldmann T, Wieghofer P, Müller PF, Wolf Y, Varol D, Yona S, et al. A new type of microglia gene targeting shows TAK1 to be pivotal in CNS autoimmune inflammation. Nat Neurosci. 2013;16:1618-26.

7. Yona S, Kim KW, Wolf Y, Mildner A, Varol D, Breker M, et al. Fate mapping reveals origins and dynamics of monocytes and tissue macrophages under homeostasis. Immunity. 2013;38:79-91.

8. Erta M, Quintana A, Hidalgo J. Interleukin-6, a major cytokine in the central nervous system. Int J Biol Sci. 2012;8:1254-66.

9. Frei K, Fredrikson S, Fontana A, Link H. Interleukin-6 is elevated in plasma in multiple sclerosis. J Neuroimmunol. 1991;31:147-53.

10. Maimone D, Guazzi GC, Annunziata P. IL-6 detection in multiple sclerosis brain. J Neurol Sci. 1997;146:59-65.

11. Eugster HP, Frei K, Kopf M, Lassmann H, Fontana A. IL-6-deficient mice resist myelin oligodendrocyte glycoprotein-induced autoimmune encephalomyelitis. Eur J Immunol. 1998:28:2178-87.

12. Giralt M, Ramos R, Quintana A, Ferrer B, Erta M, Castro-Freire M, et al. Induction of atypical EAE mediated by transgenic production of IL- 6 in astrocytes in the absence of systemic IL-6. Glia. 2013;61:587-600. 
13. Okuda Y, Sakoda S, Bernard CCA, Fujimura H, Saeki Y, Kishimoto T, et al. IL6-deficient mice are resistant to the induction of experimental autoimmune encephalomyelitis provoked by myelin oligodendrocyte glycoprotein. Int Immunol. 1998;10:703-8.

14. Samoilova EB, Horton JL, Hilliard B, Liu TT, Chen Y. IL-6-deficient mice are resistant to experimental autoimmune encephalomyelitis: roles of IL-6 in the activation and differentiation of autoreactiveT cells. J Immunol. 1998;161: 6480-6.

15. Bettelli E, Carrier Y, Gao W, Korn T, Strom TB, Oukka M, et al. Reciprocal developmental pathways for the generation of pathogenic effector $\mathrm{TH} 17$ and regulatory T cells. Nature. 2006;441:235-8.

16. Molnarfi N, Schulze-Topphoff U, Weber MS, Patarroyo JC, Prod'homme T, Varrin-Doyer M, et al. MHC class I-dependent B cell APC function is required for induction of CNS autoimmunity independent of myelin-specific antibodies. J Exp Med. 2013;210:2921-37.

17. Heink S, Yogev N, Garbers C, Herwerth M, Aly L, Gasperi C, et al. Transpresentation of IL-6 by dendritic cells is required for the priming of pathogenic TH17 cells. Nat Immunol. 2017;18:74-85.

18. Erta M, Giralt M, Jiménez S, Molinero A, Comes G, Hidalgo J. Astrocytic IL-6 influences the clinical symptoms of EAE in mice. Brain Sci. 2016;6:E15.

19. Sanchis P, Fernández-gayol O, Comes G, Escrig A, Giralt M, Palmiter RD, et al. Interleukin- 6 derived from the central nervous system may influence the pathogenesis of experimental autoimmune encephalomyelitis in a celldependent manner. Cells. 2020;9.

20. Gosselin D, Skola D, Coufal NG, Holtman IR, Schlachetzki JCM, Sajti E, et al. An environment-dependent transcriptional network specifies human microglia identity. Science. 2017;356.

21. Hanisch UK. Microglia as a source and target of cytokines. Glia. 2002;40:140-55.

22. Marik C, Felts PA, Bauer J, Lassmann H, Smith KJ. Lesion genesis in a subset of patients with multiple sclerosis: a role for innate immunity? Brain. 2007; 130:2800-15.

23. Ajami B, Bennett JL, Krieger C, McNagny KM, Rossi FM. Infiltrating monocytes trigger EAE progression, but do not contribute to the resident microglia pool. Nat Neurosci. 2011;14:1142-50

24. Farley F, Soriano P, Steffen LS, Dymecki SM. Widespread recombinase expression using FLPeR (flipper) mice. Genesis. 2000;28:106-10.

25. Sanz E, Yang L, Su T, Morris DR, McKnight GS, Amieux PS. Cell-type-specific isolation of ribosome-associated mRNA from complex tissues. Proc Natl Acad Sci U S A. 2009;106:13939-44

26. Sanchis P, Fernández-Gayol O, Vizueta J, Comes G, Canal C, Escrig A, et al. Microglial cell-derived interleukin-6 influences behavior and inflammatory response in the brain following traumatic brain injury. Glia. 2020;68:999-1016.

27. Schneider CA, Rasband WS, Eliceiri KW. NIH image of ImageJ: 25 years of Umage analysis. Nat Methods. 2012;9:671-5

28. Ruifrok AC, Johnston DA. Quantification of histochemical staining by color deconvolution. Anal Quant Cytol Histol. 2001;23:291-9.

29. Cardin JA, Carlén M, Meletis K, Knoblich U, Zhang F, Deisseroth K, et al. Driving fast-spiking cells induces gamma rhythm and controls sensory responses. Nature. 2009;459:663-7.

30. Sohal VS, Zhang F, Yizhar O, Deisseroth K. Parvalbumin neurons and gamma rhythms enhance cortical circuit performance. Nature. 2009;459:698-702.

31. Tsai H-C, Zhang F, Adamantidis A, Stuber GD, Bonci A, de Lecea L, et al. Phasic firing in dopaminergic neurons is sufficient for behavioral conditioning. Science. 2009;324:1080-4.

32. Turan S, Bode J. Site-specific recombinases: from tag-and-target- to tag-andexchange-based genomic modifications. FASEB J. 2011;25:4088-107.

33. Samokhvalov IM, Thomson AM, Lalancette C, Liakhovitskaia A, Ure J, Medvinsky A. Multifunctional reversible knockout/reporter system enabling fully functional reconstitution of the AML1/Runx1 locus and rescue of hematopoiesis. Genesis. 2006:44:115-21.

34. Guy J, Gan J, Selfridge J, Cobb S, Bird A. Reversal of neurological defects in a mouse model of Rett syndrome. Science. 2007:315:1143-7.

35. Ventura A, Kirsch DG, McLaughlin ME, Tuveson DA, Grimm J, Lintault L, et al. Restoration of p53 function leads to tumour regression in vivo. Nature. 2007;445:661-5.

36. Zhou X, Vink M, Klaver B, Berkhout B, Das AT. Optimization of the Tet-on system for regulated gene expression through viral evolution. Gene Ther. 2006;13:1382-90.

37. Heyer J, Kwong LN, Lowe SW, Chin L. Non-germline genetically engineered mouse models for translational cancer research. Nat Rev Cancer. 2010;10: 470-80.
38. Shimizu E, Tang Y-P, Rampon C, Tsien JZ. NMDA receptor-dependent synaptic reinforcement as a crucial process for memory consolidation. Science. 2000;290:1170-4.

39. Chaiyachati BH, Kaundal R, Zhao J, Wu J, Flavell R, Chi T. LoxP-FRT trap (LOFT): a simple and flexible system for conventional and reversible gene targeting. BMC Biol. 2012;10:96.

40. Kopf M, Baumannt H, Freer G, Freudenberg M, Lamers M, Kishimoto T, et al. Impaired immune and acute-phase responses in interleukin-6-deficient mice. Nature. 1994;368:339-42.

41. Mendel I, Katz A, Kozak N, Ben-Nun A, Revel M. Interleukin-6 functions in autoimmune encephalomyelitis: a study in gene-targeted mice. Eur J Immunol. 1998;28:1727-37.

42. Serada S, Fujimoto M, Mihara M, Koike N, Ohsugi Y, Nomura S, et al. IL-6 blockade inhibits the induction of myelin antigen-specific Th17 cells and Th1 cells in experimental autoimmune encephalomyelitis. Proc Natl Acad Sci U S A. 2008;105:9041-6.

43. Brod SA, Bauer VL. Ingested (oral) tocilizumab inhibits EAE. Cytokine. 2014; 68:86-93.

44. Ponomarev ED, Shriver LP, Maresz K, Dittel BN. Microglial cell activation and proliferation precedes the onset of CNS autoimmunity. J Neurosci Res. 2005; 81:374-89.

45. Murphy ÁC, Lalor SJ, Lynch MA, Mills KHG. Infiltration of Th1 and Th17 cells and activation of microglia in the CNS during the course of experimental autoimmune encephalomyelitis. Brain Behav Immun. 2010;24:641-51.

46. Jung S, Aliberti J, Graemmel P, Sunshine MJ, Kreutzberg GW, Sher A, et al. Analysis of Fractalkine receptor CX3CR1 function by targeted deletion and green fluorescent protein reporter gene insertion. Mol Cell Biol. 2000;20: 4106-14.

47. Geissmann F, Jung S, Littman DR. Blood monocytes consist of two principal subsets with distinct migratory properties. Immunity. 2003;19:71-82.

48. Valny M, Honsa P, Kirdajova D, Kamenik Z, Anderova M. Tamoxifen in the mouse brain: implications for fate-mapping studies using the tamoxifeninducible cre-loxP system. Front Cell Neurosci. 2016;10:1-12.

49. Ellisor D, Zervas M. Tamoxifen dose response and conditional cell marking: is there control? Mol Cell Neurosci. 2010;45:132-8.

50. Bebo BF, Dehghani B, Foster S, Kurniawan A, Lopez FJ, Sherman LS. Treatment with selective estrogen receptor modulators regulates myelin specific T-cells and suppresses experimental autoimmune encephalomyelitis. Glia. 2009:57:777-90.

51. Ajami B, Bennett JL, Krieger C, Tetzlaff W, Rossi FMV. Local self-renewal can sustain CNS microglia maintenance and function throughout adult life. Nat Neurosci. 2007;10:1538-43.

52. Ginhoux F, Greter M, Leboeuf M, Nandi S, See P, Gokhan S, et al. Fate mapping analysis reveals that adult microglia derive from primitive macrophages. Science. 2010;701:841-5.

53. Korn T, Bettelli E, Gao W, Awasthi A, Jäger A, Strom TB, et al. IL-21 initiates an alternative pathway to induce proinflammatory T H17 cells. Nature. 2007; 448:484-8.

54. Quintana A, Muller M, Frausto RF, Ramos R, Getts DR, Sanz E, et al. Sitespecific production of IL-6 in the central nervous system retargets and enhances the inflammatory response in experimental autoimmune encephalomyelitis. J Immunol. 2009;183:2079-88.

\section{Publisher's Note}

Springer Nature remains neutral with regard to jurisdictional claims in published maps and institutional affiliations.

Ready to submit your research? Choose BMC and benefit from:

- fast, convenient online submission

- thorough peer review by experienced researchers in your field

- rapid publication on acceptance

- support for research data, including large and complex data types

- gold Open Access which fosters wider collaboration and increased citations

- maximum visibility for your research: over $100 \mathrm{M}$ website views per year

At BMC, research is always in progress.

Learn more biomedcentral.com/submission 